\title{
A virtual element method for the acoustic vibration problem
}

\author{
Lourenço Beirão da Veiga • David Mora • \\ Gonzalo Rivera • Rodolfo Rodríguez
}

\begin{abstract}
We analyze in this paper a virtual element approximation for the acoustic vibration problem. We consider a variational formulation relying only on the fluid displacement and propose a discretization by means of $\mathrm{H}($ div $)$ virtual elements with vanishing rotor. Under standard assumptions on the meshes, we show that the resulting scheme provides a correct approximation of the spectrum and prove optimal order error estimates. With this end, we prove approximation properties of the proposed virtual elements. We also report some numerical tests supporting our theoretical results.
\end{abstract}

Keywords Virtual Element Method · rotor free H(div) elements · acoustic vibration problem · polygonal meshes · error estimates

Mathematics Subject Classification (2000) 65N30 65 N25 $\cdot 70 J 30 \cdot 76 \mathrm{M} 25$

D. Mora was partially supported by CONICYT (Chile) through FONDECYT project No. 1140791 , by DIUBB through project 151408 GI/VC and by Anillo ANANUM, ACT1118, CONICYT (Chile).

G. Rivera was partially supported by a CONICYT (Chile) fellowship.

R. Rodríguez was partially supported by BASAL project, CMM, Universidad de Chile, by Anillo ANANUM, ACT1118, CONICYT (Chile) and by Red Doctoral REDOC.CTA, MINEDUC project UCO1202 at Universidad de Concepción (Chile).

L. Beirão da Veiga

Dipartimento di Matematica e Applicazioni, Università di Milano-Bicocca, 20125 Milano, Italy.

E-mail: lourenco.beirao@unimib.it

D. Mora

GIMNAP, Departamento de Matemática, Universidad del Bío-Bío, Casilla 5-C, Concepción, Chile and Centro de Investigación en Ingeniería Matemática ( $\mathrm{CI}^{2} \mathrm{MA}$ ), Universidad de Concepción, Casilla 160-C, Concepción, Chile.

E-mail: dmora@ubiobio.cl

G. Rivera · R. Rodríguez

$\mathrm{CI}^{2} \mathrm{MA}$, Departamento de Ingeniería Matemática, Universidad de Concepción, Casilla 160-C, Concepción, Chile.

E-mail: \{grivera,rodolfo\}@ing-mat.udec.cl 


\section{Introduction}

The Virtual Element Method (VEM) introduced in [6] is a recent generalization of the Finite Element Method which is characterized by the capability of dealing with very general polygonal/polyhedral meshes and the possibility to easily implement highly regular discrete spaces. Indeed, by avoiding the explicit construction of the local basis functions, the VEM can easily handle general polygons/polyhedrons without complex integrations on the element (see [7] for details on the coding aspects of the method). The interest in numerical methods that can make use of general polytopal meshes has recently undergone a significant growth in the mathematical and engineering literature; among the large number of papers on this subject, we cite as a minimal sample [3, $9,22,26,27,35,36]$. Regarding the VEM literature, we limit ourselves to the following few articles $[1,2,4,6-8,10,11,20,21,28,32,33]$.

The numerical approximation of eigenvalue problems for partial differential equations derived from engineering applications, is object of great interest from both, the practical and theoretical points of view. We refer to $[17,18]$ and the references therein for the state of the art in this subject area. In particular, this paper focus on the so called acoustic vibration problem; namely, to compute the vibration modes and the natural frequencies of an inviscid compressible fluid within a rigid cavity [37]. One motivation for considering this problem is that it constitutes a stepping stone towards the more challenging goal of devising virtual element spectral approximations for coupled systems involving fluid-structure interaction, which arises in many engineering problems. The simplest formulation of this problem is obtained by using pressure variations which leads to an eigenvalue problem for the Laplace operator [37]. However, for coupled problems, it is convenient to use a dual formulation in terms of fluid displacements (see [30]). A standard finite element approximation of this problem leads to spurious modes (see [29]). Such a spectral pollution can be avoided by using H(div)-conforming elements, like Raviart-Thomas finite elements [12,15,16,18,34]. See [14] for a thorough discussion on this topic.

The aim of this paper is to introduce and analyze an $\mathrm{H}$ (div) VEM which applies to general polygonal (even non-convex) meshes for the two-dimensional acoustic vibration problem. We begin with a variational formulation of the spectral problem relying only on the fluid displacement. Then, we propose a discretization based on the mixed VEM introduced in [8] for general second order elliptic problems. The well-known abstract spectral approximation theory (see [5]) cannot be used to deal with the analysis of our problem. Indeed, the kernel of the bilinear form on the left-hand side of the variational formulation has in our case an infinite-dimensional kernel. Although the standard shift strategy allows a solution operator to be defined, this is not compact and its nontrivial essential spectrum may in such cases lead to spectral pollution at the discrete level. However, by appropriately adapting the abstract spectral approximation theory for non-compact operators developed in [24,25], under rather mild assumptions on the polygonal meshes, we establish that the resulting scheme provides a correct approximation of the spectrum and prove error estimates for the eigenfunctions and a double order for the eigenvalues. As a by-product, we derive optimal approximation estimates for $\mathrm{H}$ (div) virtual elements with vanishing rotor, a result that could be useful also for other applications. These results and their corresponding proofs are collected in an appendix.

The outline of this article is as follows: We introduce in Section 2 the variational formulation of the acoustic vibration problem, define a solution operator and establish 
its spectral characterization. In Section 3, we introduce the virtual element discrete formulation, describe the spectrum of a discrete solution operator and establish some auxiliary results. In Section 4, we prove that the numerical scheme provides a correct spectral approximation and establish optimal order error estimates for the eigenvalues and eigenfunctions. In Section 5, we report a couple of numerical tests that allow us to assess the convergence properties of the method, to confirm that it is not polluted with spurious modes and to check that the experimental rates of convergence agree with the theoretical ones. Finally, we introduce in an appendix the proofs of the approximation results for the introduced virtual element interpolant.

Throughout the paper, $\Omega$ is a generic Lipschitz bounded domain of $\mathbb{R}^{2}$. For $s \geq 0$, $\|\cdot\|_{s, \Omega}$ stands indistinctly for the norm of the Hilbertian Sobolev spaces $\mathrm{H}^{s}(\Omega)$ or $\left[\mathrm{H}^{s}(\Omega)\right]^{2}$ with the convention $\mathrm{H}^{0}(\Omega):=\mathrm{L}^{2}(\Omega)$. We also define the Hilbert space $\mathrm{H}(\operatorname{div} ; \Omega):=\left\{\boldsymbol{v} \in\left[\mathrm{L}^{2}(\Omega)\right]^{2}: \operatorname{div} \boldsymbol{v} \in \mathrm{L}^{2}(\Omega)\right\}$, whose norm is given by $\|\boldsymbol{v}\|_{\operatorname{div}, \Omega}^{2}:=$ $\|\boldsymbol{v}\|_{0, \Omega}^{2}+\|\operatorname{div} \boldsymbol{v}\|_{0, \Omega}^{2}$. Finally, we employ $\mathbf{0}$ to denote a generic null vector and $C$ to denote generic constants independent of the discretization parameters, which may take different values at different places.

\section{The spectral problem}

We consider the free vibration problem for an acoustic fluid within a bounded rigid cavity $\Omega \subset \mathbb{R}^{2}$ with polygonal boundary $\Gamma$ and outward unit normal vector $\boldsymbol{n}$ :

$$
\begin{cases}-\omega^{2} \varrho \boldsymbol{w}=-\nabla p & \text { in } \Omega, \\ p=-\varrho c^{2} \operatorname{div} \boldsymbol{w} & \text { in } \Omega, \\ \boldsymbol{w} \cdot \boldsymbol{n}=0 & \text { on } \Gamma,\end{cases}
$$

where $\boldsymbol{w}$ is the fluid displacement, $p$ is the pressure fluctuation, $\varrho$ the density, $c$ the acoustic speed and $\omega$ the vibration frequency. Multiplying the first equation above by a test function

$$
\boldsymbol{v} \in \mathcal{V}:=\{\boldsymbol{v} \in \mathrm{H}(\operatorname{div} ; \Omega): \boldsymbol{v} \cdot \boldsymbol{n}=0 \text { on } \Gamma\}
$$

integrating by parts, using the boundary condition and eliminating $p$, we arrive at the following weak formulation in which, for simplicity, we have taken the physical parameters $\varrho$ and $c$ equal to one and denote $\lambda=\omega^{2}$ :

Problem 1 Find $(\lambda, \boldsymbol{w}) \in \mathbb{R} \times \mathcal{V}, \boldsymbol{w} \neq 0$, such that

$$
\int_{\Omega} \operatorname{div} \boldsymbol{w} \operatorname{div} \boldsymbol{v}=\lambda \int_{\Omega} \boldsymbol{w} \cdot \boldsymbol{v} \quad \forall \boldsymbol{v} \in \mathcal{V} .
$$

Since the bilinear form on the left-hand side is not $\mathrm{H}(\operatorname{div} ; \Omega)$-elliptic, it is convenient to use a shift argument to rewrite this eigenvalue problem in the following equivalent form:

Problem 2 Find $(\lambda, \boldsymbol{w}) \in \mathbb{R} \times \mathcal{V}, \boldsymbol{w} \neq 0$, such that

$$
a(\boldsymbol{w}, \boldsymbol{v})=(\lambda+1) b(\boldsymbol{w}, \boldsymbol{v}) \quad \forall \boldsymbol{v} \in \mathcal{V}
$$


where the bilinear forms are defined for any $\boldsymbol{w}, \boldsymbol{v} \in \mathcal{V}$ by

$$
\begin{aligned}
& a(\boldsymbol{w}, \boldsymbol{v}):=\int_{\Omega} \operatorname{div} \boldsymbol{w} \operatorname{div} \boldsymbol{v}+\int_{\Omega} \boldsymbol{w} \cdot \boldsymbol{v} \\
& b(\boldsymbol{w}, \boldsymbol{v}):=\int_{\Omega} \boldsymbol{w} \cdot \boldsymbol{v} .
\end{aligned}
$$

We define the solution operator associated with Problem 2:

$$
\begin{aligned}
\mathrm{T}: \mathcal{V} & \longrightarrow \mathcal{V} \\
f & \longmapsto \mathrm{T} f:=\boldsymbol{u}
\end{aligned}
$$

where $\boldsymbol{u} \in \mathcal{V}$ is the solution of the corresponding source problem:

$$
a(\boldsymbol{u}, \boldsymbol{v})=b(\boldsymbol{f}, \boldsymbol{v}) \quad \forall \boldsymbol{v} \in \mathcal{V} .
$$

Since the bilinear form $a(\cdot, \cdot)$ is $\mathrm{H}(\operatorname{div} ; \Omega)$-elliptic, the problem above is well posed. As an immediate consequence, we deduce that the linear operator $\mathbf{T}$ is well defined and bounded. Notice that $(\lambda, \boldsymbol{w}) \in \mathbb{R} \times \mathcal{V}$ solves Problem 1 if and only if $(1 /(1+\lambda), \boldsymbol{w})$ is an eigenpair of $\mathbf{T}$, i.e, if and only if

$$
\mathbf{T} \boldsymbol{w}=\mu \boldsymbol{w}, \quad \text { with } \mu:=\frac{1}{\lambda+1} .
$$

Moreover, it is easy to check that $\mathbf{T}$ is self-adjoint with respect to the inner products $a(\cdot, \cdot)$ and $b(\cdot, \cdot)$ in $\mathcal{V}$.

In what follows, we recall some results that can be found in [12] in the more general context of fluid-solid vibration problems. The proofs in [12] can be readily adapted to this case to obtain the following results. Let the space

$$
\mathcal{K}:=\{\boldsymbol{v} \in \mathcal{V}: \operatorname{div} \boldsymbol{v}=0 \text { in } \Omega\} .
$$

Lemma 1 The operator $\mathbf{T}$ admits the eigenvalue $\mu=1$ with associated eigenspace $\mathcal{K}$.

The following result provides a simple characterization of the orthogonal complement of $\mathcal{K}$ in $\mathcal{V}$.

Lemma 2 Let $\mathcal{G}:=\left\{\nabla q: q \in \mathrm{H}^{1}(\Omega)\right\}$. Then,

$$
\mathcal{V}=\mathcal{K} \oplus(\mathcal{G} \cap \mathcal{V})
$$

is an orthogonal decomposition in both $\left[\mathrm{L}^{2}(\Omega)\right]^{2}$ and $\mathrm{H}(\operatorname{div} ; \Omega)$.

Moreover, there exists $s \in(1 / 2,1]$ such that, for all $\boldsymbol{v} \in \mathcal{V}$, if $\boldsymbol{v}=\boldsymbol{\varphi}+\nabla q$ with $\boldsymbol{\varphi} \in \mathcal{K}$ and $\nabla q \in \mathcal{G} \cap \mathcal{V}$, then $\nabla q \in\left[\mathrm{H}^{s}(\Omega)\right]^{2}$ and $\|\nabla q\|_{s, \Omega} \leq C\|\operatorname{div} \boldsymbol{v}\|_{0, \Omega}$.

From now on, we fix $s \in(1 / 2,1]$ such that the above lemma holds true.

The following result shows that the subspace $\mathcal{G} \cap \mathcal{V}$ is invariant for $\mathbf{T}$.

Lemma 3 There holds

$$
\mathbf{T}(\mathcal{G} \cap \mathcal{V}) \subset(\mathcal{G} \cap \mathcal{V})
$$

Smoothing properties of $\mathbf{T}$ as an operator from $\mathcal{G} \cap \mathcal{V}$ into itself are established in what follows. 
Theorem 1 There holds

$$
\mathbf{T}(\mathcal{G} \cap \mathcal{V}) \subset\left\{\boldsymbol{v} \in\left[\mathrm{H}^{s}(\Omega)\right]^{2}: \operatorname{div} \boldsymbol{v} \in \mathrm{H}^{1}(\Omega)\right\}
$$

and there exists $C>0$ such that, for all $\boldsymbol{f} \in \mathcal{G} \cap \mathcal{V}$, if $\boldsymbol{u}=\mathbf{T} \boldsymbol{f}$, then

$$
\|\boldsymbol{u}\|_{s, \Omega}+\|\operatorname{div} \boldsymbol{u}\|_{1, \Omega} \leq C\|\boldsymbol{f}\|_{\operatorname{div}, \Omega} .
$$

Consequently, the operator $\left.\mathbf{T}\right|_{\mathcal{G} \cap \mathcal{V}}: \mathcal{G} \cap \mathcal{V} \rightarrow \mathcal{G} \cap \mathcal{V}$ is compact.

Finally, the following result provides a spectral characterization of $\mathbf{T}$.

Theorem 2 The spectrum of $\mathbf{T}$ decomposes as $\operatorname{sp}(\mathbf{T})=\{0,1\} \cup\left\{\mu_{k}\right\}_{k \in \mathbb{N}}$, where:

i) $\mu=1$ is an infinite-multiplicity eigenvalue of $\mathbf{T}$ and its associated eigenspace is $\mathcal{K}$;

ii) $\left\{\mu_{k}\right\}_{k \in \mathbb{N}} \subset(0,1)$ is a sequence of finite-multiplicity eigenvalues of $\mathbf{T}$ which converge to 0 and if $\boldsymbol{w}$ is an eigenfunction of $\mathbf{T}$ associated with such an eigenvalue, then there exists $\tilde{s}>1 / 2$ and $C>0$, both depending on the eigenvalue, such that

$$
\|\boldsymbol{w}\|_{\tilde{s}, \Omega}+\|\operatorname{div} \boldsymbol{w}\|_{1+\tilde{s}, \Omega} \leq C\|\boldsymbol{w}\|_{\operatorname{div}, \Omega}
$$

iii) $\mu=0$ is not an eigenvalue of $\mathbf{T}$.

\section{The virtual elements discretization}

We begin this section, by recalling the mesh construction and the assumptions considered to introduce a discrete virtual element space. Then, we will introduce a virtual element discretization of Problem 1 and provide a spectral characterization of the resulting discrete eigenvalue problem. Let $\left\{\mathcal{T}_{h}\right\}$ be a family of decompositions of $\Omega$ into polygons $E$. Let $h_{E}$ denote the diameter of the element $E$ and $h:=\max _{E \in \Omega} h_{E}$.

For the analysis, we make the following assumptions on the meshes as in $[8,20]$ : there exists a positive real number $C_{\mathcal{T}}$ such that, for every $E \in \mathcal{T}_{h}$ and for every $\mathcal{T}_{h}$,

- $\mathbf{A}_{1}$ : the ratio between the shortest edge and the diameter of $E$ is larger than $C_{\mathcal{T}}$;

$-\mathbf{A}_{2}: E$ is star-shaped with respect to every point of a ball of radius $C_{\mathcal{T}} h_{E}$.

For any subset $S \subseteq \mathbb{R}^{2}$ and any non-negative integer $k$, we indicate by $\mathbb{P}_{k}(S)$ the space of polynomials of degree up to $k$ defined on $S$. To keep the notation simpler, we denote by $\boldsymbol{n}$ a generic normal unit vector; in each case, its precise definition will be clear from the context. We consider now a polygon $E$ and, for any fixed non-negative integer $k$, we define the following finite dimensional space (inspired in $[20,8]$ ):

$\mathcal{V}_{h}^{E}:=\left\{\boldsymbol{v}_{h} \in \mathrm{H}(\operatorname{div} ; E):\left(\boldsymbol{v}_{h} \cdot \boldsymbol{n}\right) \in \mathbb{P}_{k}(e) \forall e \subset \partial E, \operatorname{div} \boldsymbol{v}_{h} \in \mathbb{P}_{k}(E), \operatorname{rot} \boldsymbol{v}_{h}=0\right.$ in $\left.E\right\}$

Remark 1 It is elementary to check that a vector field $\boldsymbol{v}_{h} \in \mathcal{V}_{h}^{E}$ satisfying $\boldsymbol{v}_{h} \cdot \boldsymbol{n}=0$ on $\partial E$ and $\operatorname{div} \boldsymbol{v}_{h}=0$ in $E$ is identically zero. In fact, since a star-shaped polygon $E$ is simply connected and $\operatorname{rot} \boldsymbol{v}_{h}=0$ in $E$, there exists $\gamma \in \mathrm{H}^{1}(E)$ such that $\boldsymbol{v}_{h}=\nabla \gamma$. Then, $\Delta \gamma=\operatorname{div} \boldsymbol{v}_{h}=0$ in $E$ and $\partial \gamma / \partial \boldsymbol{n}=\boldsymbol{v}_{h} \cdot \boldsymbol{n}=0$ on $\partial E$. Hence, $\boldsymbol{v}_{h}=\nabla \gamma=\mathbf{0}$ in $E$. This implies that $\mathcal{V}_{h}^{E}$ is finite dimensional, the dimension being less or equal to $N_{E}(k+1)+(k+1)(k+2) / 2-1$, where $N_{E}$ is the number of edges of $E$. 
We define the following degrees of freedom for functions $\boldsymbol{v}_{h}$ in $\mathcal{V}_{h}^{E}$ :

$$
\begin{array}{cl}
\int_{e}\left(\boldsymbol{v}_{h} \cdot \boldsymbol{n}\right) q d s & \forall q \in \mathbb{P}_{k}(e), \quad \forall \text { edge } e \subset \partial E ; \\
\int_{E} \boldsymbol{v}_{h} \cdot \nabla q & \forall q \in \mathbb{P}_{k}(E) / \mathbb{P}_{0}(E) .
\end{array}
$$

Proposition 1 The degrees of freedom (1)-(2) are unisolvent in $\mathcal{V}_{h}^{E}$.

Proof It is easy to check that the number of degrees of freedom (1)-(2) equals the dimension of $\mathcal{V}_{h}^{E}$. Thus, we only need to show that if $\boldsymbol{v}_{h}$ in $\mathcal{V}_{h}^{E}$ is such that

$$
\begin{array}{cl}
\int_{e}\left(\boldsymbol{v}_{h} \cdot \boldsymbol{n}\right) q d s=0 & \forall q \in \mathbb{P}_{k}(e), \quad \forall \text { edge } e \subset \partial E \\
\int_{E} \boldsymbol{v}_{h} \cdot \nabla q=0 & \forall q \in \mathbb{P}_{k}(E) / \mathbb{P}_{0}(E),
\end{array}
$$

then $\boldsymbol{v}_{h}=\mathbf{0}$. Since $\operatorname{div} \boldsymbol{v}_{h} \in \mathbb{P}_{k}(E)$, by taking $q:=\operatorname{div} \boldsymbol{v}_{h}$ above, we have

$$
\int_{E}\left(\operatorname{div} \boldsymbol{v}_{h}\right)^{2}=\int_{E} \operatorname{div} \boldsymbol{v}_{h} q=-\int_{E} \boldsymbol{v}_{h} \cdot \nabla q+\int_{\partial E}\left(\boldsymbol{v}_{h} \cdot \boldsymbol{n}\right) q d s=0 .
$$

Then, div $\boldsymbol{v}_{h}=0$. Similarly, for each edge $e \subset \partial E$, since $\boldsymbol{v}_{h} \cdot \boldsymbol{n} \in \mathbb{P}_{k}(e)$, by taking $q:=\boldsymbol{v}_{h} \cdot \boldsymbol{n}$ we obtain

$$
\int_{e}\left(\boldsymbol{v}_{h} \cdot \boldsymbol{n}\right)^{2} d s=0
$$

Hence, $\boldsymbol{v}_{h} \cdot \boldsymbol{n}=0$ on $\partial E$. Therefore, according to Remark $1, \boldsymbol{v}_{h}=\mathbf{0}$ in $E$.

Remark 2 For the degrees of freedom (2), we could integrate by parts and substitute them with

$$
\int_{E} \operatorname{div} \boldsymbol{v}_{h} q \quad \forall q \in \mathbb{P}_{k}(E) / \mathbb{P}_{0}(E) .
$$

Needless to say, certain degrees of freedom will be more convenient when writing the code and the others might be more convenient when writing a proof.

For each decomposition $\mathcal{T}_{h}$ of $\Omega$ into polygons $E$, we define

$$
\mathcal{V}_{h}:=\left\{\boldsymbol{v}_{h} \in \mathcal{V}:\left.\boldsymbol{v}_{h}\right|_{E} \in \mathcal{V}_{h}^{E}\right\}
$$

In agreement with the local choice, we choose the following global degrees of freedom:

$$
\begin{aligned}
\int_{e}\left(\boldsymbol{v}_{h} \cdot \boldsymbol{n}\right) q d s & \forall q \in \mathbb{P}_{k}(e), \quad \text { for each internal edge } e \not \subset \Gamma ; \\
\int_{E} \boldsymbol{v}_{h} \cdot \nabla q & \forall q \in \mathbb{P}_{k}(E) / \mathbb{P}_{0}(E), \quad \text { for each element } E \in \mathcal{T}_{h} .
\end{aligned}
$$


Remark 3 The number of internal degrees of freedom of the Virtual Element Method here considered $\left(V E M_{k}\right)$ is in general less than that of standard finite elements of the same order such as Raviart-Thomas $\left(R T_{k}\right)$ or Brezzi-Douglas-Marini $\left(B D M_{k}\right)$ elements, while the number of degrees of freedom per edge is the same. A count of the internal degrees of freedom gives

$$
R T_{k}: k(k+1), \quad B D M_{k}:(k+1)(k-1), \quad V E M_{k}:(k+1)(k+2) / 2-1 .
$$

The proposed family may therefore be preferable to more standard finite elements even in the case of triangular meshes, especially for moderate-to-high values of $k$.

In order to construct the discrete scheme, we need some preliminary definitions. First, we split the bilinear form $a(\cdot, \cdot)$ introduced in the previous section as follows:

$$
a\left(\boldsymbol{u}_{h}, \boldsymbol{v}_{h}\right)=\sum_{E \in \mathcal{T}_{h}}\left(\int_{E} \operatorname{div} \boldsymbol{u}_{h} \operatorname{div} \boldsymbol{v}_{h}+\int_{E} \boldsymbol{u}_{h} \cdot \boldsymbol{v}_{h}\right), \quad \boldsymbol{u}_{h}, \boldsymbol{v}_{h} \in \mathcal{V}_{h}
$$

The local matrices associated with the first term on the right hand side above are easily computable since $\operatorname{div} \boldsymbol{u}_{h}$ and $\operatorname{div} \boldsymbol{v}_{h}$ are polynomials in each element. We explicitly point out that, as can be seem from (1)-(2), the divergence of any vector $\boldsymbol{v}_{h} \in \mathcal{V}_{h}$ can be easily computed from knowledge of the degrees of freedom of $\boldsymbol{v}_{h}$. Instead, for the local matrices associated with the second term on the right hand side above, we must take into account that, due to the implicit space definition, it is not possible to compute exactly the integrals. Because of this, we will use an approximation of them. The final output will be a local matrix on each element $E$ whose associated bilinear form is exact whenever one of the two entries is a gradient of a polynomial of degree $k+1$. This will allow us to retain the optimal approximation properties of the space $\mathcal{V}_{h}$. With this aim, we define first for each element $E$ the space

$$
\widehat{\mathcal{V}}_{h}^{E}:=\nabla\left(\mathbb{P}_{k+1}(E)\right) \subset \mathcal{V}_{h}^{E}
$$

Then, we define the $\left[\mathrm{L}^{2}(E)\right]^{2}$-orthogonal projector $\boldsymbol{\Pi}_{h}^{E}:\left[\mathrm{L}^{2}(E)\right]^{2} \longrightarrow \widehat{\mathcal{V}}_{h}^{E}$ by

$$
\int_{E} \boldsymbol{\Pi}_{h}^{E} \boldsymbol{v} \cdot \widehat{\boldsymbol{u}}_{h}=\int_{E} \boldsymbol{v} \cdot \widehat{\boldsymbol{u}}_{h} \quad \forall \widehat{\boldsymbol{u}}_{h} \in \widehat{\mathcal{V}}_{h}^{E}
$$

We point out that $\boldsymbol{\Pi}_{h}^{E} \boldsymbol{v}_{h}$ is explicitly computable for every $\boldsymbol{v}_{h} \in \mathcal{V}_{h}^{E}$ using only its degrees of freedom (1)-(2). In fact, it is easy to check that for all $\boldsymbol{v}_{h} \in \mathcal{V}_{h}^{E}$ and for all $q \in \mathbb{P}_{k+1}(E)$,

$$
\int_{E} \boldsymbol{\Pi}_{h}^{E} \boldsymbol{v}_{h} \cdot \nabla q=\int_{E} \boldsymbol{v}_{h} \cdot \nabla q=-\int_{E} \operatorname{div} \boldsymbol{v}_{h} q+\int_{\partial E}\left(\boldsymbol{v}_{h} \cdot \boldsymbol{n}\right) q d s .
$$

Remark 4 In particular, for $k=0$, for all $\boldsymbol{v}_{h} \in \mathcal{V}_{h}^{E}$ and for all $q \in \mathbb{P}_{1}(E)$, we have that

$$
\int_{E} \boldsymbol{\Pi}_{h}^{E} \boldsymbol{v}_{h} \cdot \nabla q=-\left(\frac{1}{|E|} \sum_{e \subset \partial E} \int_{e} \boldsymbol{v}_{h} \cdot \boldsymbol{n} d s\right)\left(\int_{E} q\right)+\sum_{e \subset \partial E} \int_{e}\left(\boldsymbol{v}_{h} \cdot \boldsymbol{n}\right) q d s
$$


On the other hand, let $S^{E}(\cdot, \cdot)$ be any symmetric positive definite (and computable) bilinear form to be chosen as to satisfy

$$
c_{0} \int_{E} \boldsymbol{v}_{h} \cdot \boldsymbol{v}_{h} \leq S^{E}\left(\boldsymbol{v}_{h}, \boldsymbol{v}_{h}\right) \leq c_{1} \int_{E} \boldsymbol{v}_{h} \cdot \boldsymbol{v}_{h} \quad \forall \boldsymbol{v}_{h} \in \mathcal{V}_{h}^{E},
$$

for some positive constants $c_{0}$ and $c_{1}$ depending only on the constant $C_{\mathcal{T}}$ from mesh assumptions $\mathbf{A}_{\mathbf{1}}$ and $\mathbf{A}_{\mathbf{2}}$. Then, we define on each element $E$ the bilinear form

$$
b_{h}^{E}\left(\boldsymbol{u}_{h}, \boldsymbol{v}_{h}\right):=\int_{E} \boldsymbol{\Pi}_{h}^{E} \boldsymbol{u}_{h} \cdot \boldsymbol{\Pi}_{h}^{E} \boldsymbol{v}_{h}+S^{E}\left(\boldsymbol{u}_{h}-\boldsymbol{\Pi}_{h}^{E} \boldsymbol{u}_{h}, \boldsymbol{v}_{h}-\boldsymbol{\Pi}_{h}^{E} \boldsymbol{v}_{h}\right), \quad \boldsymbol{u}_{h}, \boldsymbol{v}_{h} \in \mathcal{V}_{h}^{E}
$$

and, in a natural way,

$$
b_{h}\left(\boldsymbol{u}_{h}, \boldsymbol{v}_{h}\right):=\sum_{E \in \mathcal{T}_{h}} b_{h}^{E}\left(\boldsymbol{u}_{h}, \boldsymbol{v}_{h}\right), \quad \boldsymbol{u}_{h}, \boldsymbol{v}_{h} \in \mathcal{V}_{h}
$$

The following two properties of the bilinear form $b_{h}^{E}(\cdot, \cdot)$ are easily derived by repeating in our case the arguments from [20, Proposition 4.1].

- Consistency:

$$
b_{h}^{E}\left(\widehat{\boldsymbol{u}}_{h}, \boldsymbol{v}_{h}\right)=\int_{E} \widehat{\boldsymbol{u}}_{h} \cdot \boldsymbol{v}_{h} \quad \forall \widehat{\boldsymbol{u}}_{h} \in \widehat{\mathcal{V}}_{h}^{E}, \quad \forall \boldsymbol{v}_{h} \in \mathcal{V}_{h}^{E}, \quad \forall E \in \mathcal{T}_{h} .
$$

- Stability: There exist two positive constants $\alpha_{*}$ and $\alpha^{*}$, independent of $E$, such that:

$$
\alpha_{*} \int_{E} \boldsymbol{v}_{h} \cdot \boldsymbol{v}_{h} \leq b_{h}^{E}\left(\boldsymbol{v}_{h}, \boldsymbol{v}_{h}\right) \leq \alpha^{*} \int_{E} \boldsymbol{v}_{h} \cdot \boldsymbol{v}_{h} \quad \forall \boldsymbol{v}_{h} \in \mathcal{V}_{h}^{E}, \quad \forall E \in \mathcal{T}_{h} .
$$

Now, we are in a position to write the virtual element discretization of Problem 1.

Problem 3 Find $\left(\lambda_{h}, \boldsymbol{w}_{h}\right) \in \mathbb{R} \times \mathcal{V}_{h}, \boldsymbol{w}_{h} \neq 0$, such that

$$
\int_{\Omega} \operatorname{div} \boldsymbol{w}_{h} \operatorname{div} \boldsymbol{v}_{h}=\lambda_{h} b_{h}\left(\boldsymbol{w}_{h}, \boldsymbol{v}_{h}\right) \quad \forall \boldsymbol{v}_{h} \in \mathcal{V}_{h}
$$

We use again a shift argument to rewrite this discrete eigenvalue problem in the following convenient equivalent form.

Problem 4 Find $\left(\lambda_{h}, \boldsymbol{w}_{h}\right) \in \mathbb{R} \times \mathcal{V}_{h}, \boldsymbol{w}_{h} \neq 0$, such that

$$
a_{h}\left(\boldsymbol{w}_{h}, \boldsymbol{v}_{h}\right)=\left(\lambda_{h}+1\right) b_{h}\left(\boldsymbol{w}_{h}, \boldsymbol{v}_{h}\right) \quad \forall \boldsymbol{v}_{h} \in \mathcal{V}_{h}
$$

where

$$
a_{h}\left(\boldsymbol{w}_{h}, \boldsymbol{v}_{h}\right):=\int_{\Omega} \operatorname{div} \boldsymbol{w}_{h} \operatorname{div} \boldsymbol{v}_{h}+b_{h}\left(\boldsymbol{w}_{h}, \boldsymbol{v}_{h}\right) \quad \forall \boldsymbol{w}_{h}, \boldsymbol{v}_{h} \in \mathcal{V}_{h}
$$

We observe that by virtue of $(7)$, the bilinear form $a_{h}(\cdot, \cdot)$ is bounded. Moreover, as is shown in the following lemma, it is also uniformly elliptic.

Lemma 4 There exists a constant $\beta>0$, independent of $h$, such that

$$
a_{h}\left(\boldsymbol{v}_{h}, \boldsymbol{v}_{h}\right) \geq \beta\left\|\boldsymbol{v}_{h}\right\|_{\operatorname{div}, \Omega}^{2} \quad \forall \boldsymbol{v}_{h} \in \mathcal{V}_{h} .
$$

Proof Thanks to (7), the above inequality holds with $\beta:=\min \left\{\alpha_{*}, 1\right\}$. 
The next step is to introduce the discrete version of the operator $\mathbf{T}$ :

$$
\begin{aligned}
\mathbf{T}_{h}: & \mathcal{V}_{h} \longrightarrow \mathcal{V}_{h}, \\
\boldsymbol{f}_{h} & \longmapsto \mathbf{T}_{h} \boldsymbol{f}_{h}:=\boldsymbol{u}_{h},
\end{aligned}
$$

where $\boldsymbol{u}_{h} \in \mathcal{V}_{h}$ is the solution of the corresponding discrete source problem:

$$
a_{h}\left(\boldsymbol{u}_{h}, \boldsymbol{v}_{h}\right)=b_{h}\left(\boldsymbol{f}_{h}, \boldsymbol{v}_{h}\right) \quad \forall \boldsymbol{v}_{h} \in \mathcal{V}_{h}
$$

We deduce from Lemma 4, (7) and the Lax-Milgram Theorem, that the linear operator $\mathbf{T}_{h}$ is well defined and bounded uniformly with respect to $h$.

Once more, as in the continuous case, $\left(\lambda_{h}, \boldsymbol{w}_{h}\right)$ solves Problem 3 if and only if $\left(1 /\left(1+\lambda_{h}\right), \boldsymbol{w}_{h}\right)$ is an eigenpair of $\mathbf{T}_{h}$, i.e, if and only if

$$
\mathbf{T}_{h} \boldsymbol{w}_{h}=\mu_{h} \boldsymbol{w}_{h}, \quad \text { with } \mu_{h}:=\frac{1}{\lambda_{h}+1}
$$

Moreover, it is easy to check that $\mathbf{T}_{h}$ is self-adjoint with respect to $a_{h}(\cdot, \cdot)$ and $b_{h}(\cdot, \cdot)$. To describe the spectrum of this operator, we proceed as in the continuous case and decompose $\mathcal{V}_{h}$ into a convenient direct sum. To this end, we define

$$
\mathcal{K}_{h}:=\mathcal{V}_{h} \cap \mathcal{K}=\left\{\boldsymbol{v}_{h} \in \mathcal{V}_{h}: \operatorname{div} \boldsymbol{v}_{h}=0 \text { in } \Omega\right\}
$$

and notice that, here again, $\left.\mathbf{T}_{h}\right|_{\mathcal{K}_{h}}: \mathcal{K}_{h} \longrightarrow \mathcal{K}_{h}$ reduces to the identity. Moreover, we have the following result.

Proposition $2 \mu_{h}=1$ is an eigenvalue of $\mathbf{T}_{h}$ and its eigenspace is $\mathcal{K}_{h}$.

Proof We have that $\boldsymbol{w}_{h} \in \mathcal{V}_{h}$ is an eigenfunction associated with the eigenvalue $\mu_{h}=1$ if and only if $\int_{E} \operatorname{div} \boldsymbol{w}_{h} \operatorname{div} \boldsymbol{v}_{h}=0 \forall \boldsymbol{v}_{h} \in \mathcal{V}_{h}$, namely, if and only if $\boldsymbol{w}_{h} \in \mathcal{K}_{h}$.

As a consequence of all this, we have the following spectral characterization of the discrete solution operator.

Theorem 3 The spectrum of $\mathbf{T}_{h}$ consists of $M_{h}:=\operatorname{dim}\left(\mathcal{V}_{h}\right)$ eigenvalues, repeated according to their respective multiplicities. It decomposes as $\operatorname{sp}\left(\mathbf{T}_{h}\right)=\{1\} \cup\left\{\mu_{h k}\right\}_{k=1}^{N_{h}}$, where:

i) the eigenspace associated with $\mu_{h}=1$ is $\mathcal{K}_{h}$;

ii) $\mu_{h k} \in(0,1), k=1, \ldots, N_{h}:=M_{h}-\operatorname{dim}\left(\mathcal{K}_{h}\right)$, are non-defective eigenvalues repeated according to their respective multiplicities.

In what follows, we derive several auxiliary results which will be used in the following section to prove convergence and error estimates for the spectral approximation.

First, we establish interpolation properties in the discrete space $\mathcal{V}_{h}$. Although the $\mathcal{V}_{h}$-interpolant can be defined for less regular functions, in our case it is enough to consider $\boldsymbol{v} \in \mathcal{V}$ such that $\left.\boldsymbol{v}\right|_{E} \in\left[\mathrm{H}^{t}(E)\right]^{2}$ for some $t>1 / 2$ and for all $E \in \mathcal{T}_{h}$, so that we can easily take its trace on each individual edge. Then, we define its interpolant $\boldsymbol{v}_{I} \in \mathcal{V}_{h}$ by fixing its degrees of freedom as follows:

$$
\begin{array}{cl}
\int_{e}\left(\boldsymbol{v}-\boldsymbol{v}_{I}\right) \cdot \boldsymbol{n} q d s=0 & \forall q \in \mathbb{P}_{k}(e), \quad \forall \text { internal edge } e \not \subset \Gamma ; \\
\int_{E}\left(\boldsymbol{v}-\boldsymbol{v}_{I}\right) \cdot \nabla q=0 & \forall q \in \mathbb{P}_{k}(E) / \mathbb{P}_{0}(E), \quad \forall E \in \mathcal{T}_{h} .
\end{array}
$$


In what follows, we state two results about the approximation properties of this interpolant, whose proof we postpone to the Appendix. The first one concerns approximation properties of $\operatorname{div} \boldsymbol{v}_{I}$ and follows from a commuting diagram property for this interpolant, which involves the $\mathrm{L}^{2}(\Omega)$-orthogonal projection

$$
P_{k}: \mathrm{L}^{2}(\Omega) \longrightarrow\left\{q \in \mathrm{L}^{2}(\Omega):\left.q\right|_{E} \in \mathbb{P}_{k}(E) \quad \forall E \in \mathcal{T}_{h}\right\}
$$

Lemma 5 Let $\boldsymbol{v} \in \mathcal{V}$ be such that $\boldsymbol{v} \in\left[\mathrm{H}^{t}(\Omega)\right]^{2}$ with $t>1 / 2$. Let $\boldsymbol{v}_{I} \in \mathcal{V}_{h}$ be its interpolant defined by (8)-(9). Then,

$$
\operatorname{div} \boldsymbol{v}_{I}=P_{k}(\operatorname{div} \boldsymbol{v}) \quad \text { in } \Omega
$$

Consequently, for all $E \in \mathcal{T}_{h},\left\|\operatorname{div} \boldsymbol{v}_{I}\right\|_{0, E} \leq\|\operatorname{div} \boldsymbol{v}\|_{0, E}$ and, if $\left.\operatorname{div} \boldsymbol{v}\right|_{E} \in \mathrm{H}^{r}(E)$ with $r \geq 0$, then

$$
\left\|\operatorname{div} \boldsymbol{v}-\operatorname{div} \boldsymbol{v}_{I}\right\|_{0, E} \leq C h_{E}^{\min \{r, k+1\}}|\operatorname{div} \boldsymbol{v}|_{r, E} .
$$

The second result concerns the $\mathrm{L}^{2}(\Omega)$ approximation property of $\boldsymbol{v}_{I}$.

Lemma 6 Let $\boldsymbol{v} \in \mathcal{V}$ be such that $\boldsymbol{v} \in\left[\mathrm{H}^{t}(\Omega)\right]^{2}$ with $t>1 / 2$. Let $\boldsymbol{v}_{I} \in \mathcal{V}_{h}$ be its interpolant defined by (8)-(9). Let $E \in \mathcal{T}_{h}$. If $1 \leq t \leq k+1$, then

$$
\left\|\boldsymbol{v}-\boldsymbol{v}_{I}\right\|_{0, E} \leq C h_{E}^{t}|\boldsymbol{v}|_{t, E},
$$

whereas, if $1 / 2<t \leq 1$, then

$$
\left\|\boldsymbol{v}-\boldsymbol{v}_{I}\right\|_{0, E} \leq C\left(h_{E}^{t}|\boldsymbol{v}|_{t, E}+h_{E}\|\operatorname{div} \boldsymbol{v}\|_{0, E}\right)
$$

Let $\mathcal{K}_{h}^{\perp}$ be the $\left[\mathrm{L}^{2}(\Omega)\right]^{2}$-orthogonal complement of $\mathcal{K}_{h}$ in $\mathcal{V}_{h}$, namely,

$$
\mathcal{K}_{h}^{\perp}:=\left\{\boldsymbol{v}_{h} \in \mathcal{V}_{h}: \int_{\Omega} \boldsymbol{v}_{h} \cdot \boldsymbol{\xi}_{h}=0 \quad \forall \boldsymbol{\xi}_{h} \in \mathcal{K}_{h}\right\} .
$$

Note that $\mathcal{K}_{h}$ and $\mathcal{K}_{h}^{\perp}$ are also orthogonal in $\mathrm{H}(\operatorname{div} ; \Omega)$. The following lemma shows that, although $\mathcal{K}_{h}^{\perp} \not \subset \mathcal{K}^{\perp}=\mathcal{G} \cap \mathcal{V}$, the gradient part in the Helmholtz decomposition of a function in $\mathcal{K}_{h}^{\perp}$ is asymptotically small.

Lemma 7 Let $\boldsymbol{v}_{h} \in \mathcal{K}_{h}^{\perp}$. Then, there exist $p \in \mathrm{H}^{1+s}(\Omega)$ with $s \in(1 / 2,1]$ as in Lemma 2 and $\boldsymbol{\psi} \in \mathcal{K}$ such that $\boldsymbol{v}_{h}=\boldsymbol{\psi}+\nabla p$ and

$$
\begin{aligned}
\|\nabla p\|_{s, \Omega} & \leq C\left\|\operatorname{div} \boldsymbol{v}_{h}\right\|_{0, \Omega}, \\
\|\boldsymbol{\psi}\|_{0, \Omega} & \leq C h^{s}\left\|\operatorname{div} \boldsymbol{v}_{h}\right\|_{0, \Omega} .
\end{aligned}
$$

Proof Let $\boldsymbol{v}_{h} \in \mathcal{K}_{h}^{\perp} \subset \mathcal{V}_{h} \subset \mathcal{V}$. As a consequence of Lemma 2, we know that there exist $p \in \mathrm{H}^{1+s}(\Omega)$ and $\boldsymbol{\psi} \in \mathcal{K}$ such that $\boldsymbol{v}_{h}=\nabla p+\boldsymbol{\psi}$ and that $\|\nabla p\|_{s, \Omega} \leq C\left\|\operatorname{div} \boldsymbol{v}_{h}\right\|_{0, \Omega}$, which proves (10).

On the other hand, we have that

$$
\|\boldsymbol{\psi}\|_{0, \Omega}^{2}=\int_{\Omega}\left(\nabla p-\boldsymbol{v}_{h}\right) \cdot\left(\nabla p-(\nabla p)_{I}\right)+\int_{\Omega}\left(\nabla p-\boldsymbol{v}_{h}\right) \cdot\left((\nabla p)_{I}-\boldsymbol{v}_{h}\right) .
$$


Now, according to Lemma $5, \operatorname{div}\left((\nabla p)_{I}\right)=P_{k}(\operatorname{div}(\nabla p))$. Therefore, since $\Delta p=\operatorname{div} \boldsymbol{v}_{h}$, we obtain

$$
\operatorname{div}\left((\nabla p)_{I}-\boldsymbol{v}_{h}\right)=P_{k}(\Delta p)-\operatorname{div} \boldsymbol{v}_{h}=P_{k}\left(\operatorname{div} \boldsymbol{v}_{h}\right)-\operatorname{div} \boldsymbol{v}_{h}=0
$$

where we have used that for $\boldsymbol{v}_{h} \in \mathcal{V}_{h}$, div $\left.\boldsymbol{v}_{h}\right|_{E} \in \mathbb{P}_{k}(E)$. Therefore $\left((\nabla p)_{I}-\boldsymbol{v}_{h}\right) \in$ $\mathcal{K}_{h} \subseteq \mathcal{K}$ and since $\nabla p \in \mathcal{G} \cap \mathcal{V}=\mathcal{K}^{\perp}$ and $\boldsymbol{v}_{h} \in \mathcal{K}_{h}^{\perp}$, we have that

$$
\int_{\Omega}\left(\nabla p-\boldsymbol{v}_{h}\right) \cdot\left((\nabla p)_{I}-\boldsymbol{v}_{h}\right)=0 .
$$

Thus,

$$
\|\boldsymbol{\psi}\|_{0, \Omega}^{2}=\int_{\Omega}\left(\nabla p-\boldsymbol{v}_{h}\right) \cdot\left(\nabla p-(\nabla p)_{I}\right)
$$

and, by using Cauchy-Schwarz inequality, Lemma 6 and (10), we obtain

$$
\begin{aligned}
\|\boldsymbol{\psi}\|_{0, \Omega}^{2} & \leq \sum_{E \in \mathcal{T}_{h}}\left\|\nabla p-\boldsymbol{v}_{h}\right\|_{0, E}\left\|\nabla p-(\nabla p)_{I}\right\|_{0, E} \\
& \leq C \sum_{E \in \mathcal{T}_{h}}\left\|\nabla p-\boldsymbol{v}_{h}\right\|_{0, E}\left(h_{E}^{s}\|\nabla p\|_{s, E}+h_{E}\|\operatorname{div}(\nabla p)\|_{0, E}\right) \\
& \leq C h^{s}\|\boldsymbol{\psi}\|_{0, \Omega}\left\|\operatorname{div} \boldsymbol{v}_{h}\right\|_{0, \Omega},
\end{aligned}
$$

which allows us to complete the proof.

To end this section, we prove the following result which will be used in the sequel. Let $\boldsymbol{\Pi}_{h}$ be defined in $\mathcal{V}$ by

$$
\left.\left(\boldsymbol{\Pi}_{h} \boldsymbol{v}\right)\right|_{E}:=\boldsymbol{\Pi}_{h}^{E}\left(\left.\boldsymbol{v}\right|_{E}\right) \quad \text { for all } E \in \mathcal{T}_{h}
$$

with $\boldsymbol{\Pi}_{h}^{E}$ defined by (3).

Lemma 8 There exists a constant $C>0$ such that, for every $p \in \mathrm{H}^{1+t}(\Omega)$ with $1 / 2<$ $t \leq k+1$, there holds

$$
\left\|\nabla p-\Pi_{h}(\nabla p)\right\|_{0, \Omega} \leq C h^{t}\|\nabla p\|_{t, \Omega}
$$

Proof The result follows from the fact that, since $\boldsymbol{\Pi}_{h}^{E}$ is the $\left[\mathrm{L}^{2}(E)\right]^{2}$-projection onto $\widehat{\mathcal{V}}_{h}^{E}:=\nabla\left(\mathbb{P}_{k+1}(E)\right)(\mathrm{cf} .(3))$,

$$
\left\|\nabla p-\boldsymbol{\Pi}_{h}^{E}(\nabla p)\right\|_{0, E}=\inf _{q \in \mathbb{P}_{k+1}(E)}\|\nabla p-\nabla q\|_{0, E} \leq C h_{E}^{t}\|\nabla p\|_{t, E}
$$

Let us remark that the last inequality is a consequence of standard approximation estimates for polynomials on polygons in case of integer $t$ (see, for instance, [19, Lemma 4.3.8]) and standard Banach space interpolation results for non-integer $t$. 


\section{Spectral approximation and error estimates}

To prove that $\mathbf{T}_{h}$ provides a correct spectral approximation of $\mathbf{T}$, we will resort to the theory developed in [24] for non-compact operators. To this end, we first introduce some notation. For any linear bounded operator $\mathbf{S}: \mathcal{V} \longrightarrow \mathcal{V}$, we define

$$
\|\mathbf{S}\|_{h}:=\sup _{\mathbf{0} \neq \boldsymbol{v}_{h} \in \mathcal{V}_{h}} \frac{\left\|\mathbf{S} \boldsymbol{v}_{h}\right\|_{\operatorname{div}, \Omega}}{\left\|\boldsymbol{v}_{h}\right\|_{\operatorname{div}, \Omega}}
$$

We recall the definition of the gap $\widehat{\delta}$ between two closed subspaces $\mathcal{X}$ and $\mathcal{Y}$ of $\mathcal{V}$ :

$$
\widehat{\delta}(\mathcal{X}, \mathcal{Y}):=\max \{\delta(\mathcal{X}, \mathcal{Y}), \delta(\mathcal{Y}, \mathcal{X})\}
$$

where

$$
\delta(\mathcal{X}, \mathcal{Y}):=\sup _{\substack{\boldsymbol{x} \in \mathcal{X} \\\|\boldsymbol{x}\|_{\mathrm{div}, \Omega}=1}} \delta(\boldsymbol{x}, \mathcal{Y}) \quad \text { with } \delta(\boldsymbol{x}, \mathcal{Y}):=\inf _{\boldsymbol{y} \in \mathcal{Y}}\|\boldsymbol{x}-\boldsymbol{y}\|_{\operatorname{div}, \Omega}
$$

The theory from [24] guarantees approximation of the spectrum of $\mathbf{T}$, provided the following two properties are satisfied:

- P1: $\left\|\mathbf{T}-\mathbf{T}_{h}\right\|_{h} \rightarrow 0$ as $h \rightarrow 0$;

- P2: $\forall \boldsymbol{v} \in \mathcal{V} \quad \lim _{h \rightarrow 0} \delta\left(\boldsymbol{v}, \mathcal{V}_{h}\right)=0$

Property $\mathbf{P 2}$ follows immediately from the density of the smooth functions in $\mathcal{V}$ and the approximation properties in Lemmas 5 and 6 . Hence, there only remains to prove property $\mathbf{P 1}$. With this aim, first we note that since $\left.\mathbf{T}\right|_{\mathcal{K}_{h}}$ and $\left.\mathbf{T}_{h}\right|_{\mathcal{K}_{h}}$ both reduce to the identity, it is enough to estimate $\left\|\left(\mathbf{T}-\mathbf{T}_{h}\right) \boldsymbol{f}_{h}\right\|_{\operatorname{div}, \Omega}$ for $\boldsymbol{f}_{h} \in \mathcal{K}_{h}^{\perp}$.

Lemma 9 There exists $C>0$ such that, for all $\boldsymbol{f}_{h} \in \mathcal{K}_{h}^{\perp}$,

$$
\left\|\left(\mathbf{T}-\mathbf{T}_{h}\right) \boldsymbol{f}_{h}\right\|_{\operatorname{div}, \Omega} \leq C h^{s}\left\|\boldsymbol{f}_{h}\right\|_{\operatorname{div}, \Omega}
$$

with $s \in(1 / 2,1]$ as in Lemma 2.

Proof Let $\boldsymbol{f}_{h} \in \mathcal{K}_{h}^{\perp}, \boldsymbol{u}:=\mathbf{T} \boldsymbol{f}_{h}$ and $\boldsymbol{u}_{h}:=\mathbf{T}_{h} \boldsymbol{f}_{h}$. According to Lemma 2, we write $\boldsymbol{u}=\boldsymbol{\varphi}+\nabla q$ with $\boldsymbol{\varphi} \in \mathcal{K}, \nabla q \in\left[\mathrm{H}^{s}(\Omega)\right]^{2}$ and $\|\nabla q\|_{s, \Omega} \leq C\|\operatorname{div} \boldsymbol{u}\|_{0, \Omega}$. We have

$$
\left\|\left(\mathbf{T}-\mathbf{T}_{h}\right) \boldsymbol{f}_{h}\right\|_{\operatorname{div}, \Omega} \leq\left\|\boldsymbol{u}-(\nabla q)_{I}\right\|_{\operatorname{div}, \Omega}+\left\|\boldsymbol{u}_{h}-(\nabla q)_{I}\right\|_{\operatorname{div}, \Omega},
$$

where $(\nabla q)_{I}$ is the $\mathcal{V}_{h}$-interpolant of $\nabla q$ defined by $(8)-(9)$. We define $\boldsymbol{v}_{h}:=\boldsymbol{u}_{h}-$ $(\nabla q)_{I} \in \mathcal{V}_{h}$. Thanks to Lemma 4, the definition (5) of $b_{h}^{E}(\cdot, \cdot)$ and those of $\mathbf{T}$ and $\mathbf{T}_{h}$, we have

$$
\begin{aligned}
\beta\left\|\boldsymbol{v}_{h}\right\|_{\operatorname{div}, \Omega}^{2} \leq & a_{h}\left(\boldsymbol{v}_{h}, \boldsymbol{v}_{h}\right)=a_{h}\left(\boldsymbol{u}_{h}, \boldsymbol{v}_{h}\right)-a_{h}\left((\nabla q)_{I}, \boldsymbol{v}_{h}\right) \\
= & b_{h}\left(\boldsymbol{f}_{h}, \boldsymbol{v}_{h}\right)-\int_{\Omega} \operatorname{div}\left((\nabla q)_{I}\right) \operatorname{div} \boldsymbol{v}_{h}-\sum_{E \in \mathcal{T}_{h}} b_{h}^{E}\left((\nabla q)_{I}, \boldsymbol{v}_{h}\right) \\
= & b_{h}\left(\boldsymbol{f}_{h}, \boldsymbol{v}_{h}\right)-\int_{\Omega} \boldsymbol{f}_{h} \cdot \boldsymbol{v}_{h}+\int_{\Omega} \operatorname{div}\left(\boldsymbol{u}-(\nabla q)_{I}\right) \operatorname{div} \boldsymbol{v}_{h} \\
& -\sum_{E \in \mathcal{T}_{h}}\left(b_{h}^{E}\left((\nabla q)_{I}-\boldsymbol{\Pi}_{h}^{E} \boldsymbol{u}, \boldsymbol{v}_{h}\right)+\int_{E}\left(\boldsymbol{\Pi}_{h}^{E} \boldsymbol{u}-\boldsymbol{u}\right) \cdot \boldsymbol{v}_{h}\right),
\end{aligned}
$$


where for the last equality we have also used the consistency property (6). Since $\operatorname{div}\left((\nabla q)_{I}\right)=P_{k}(\operatorname{div}(\nabla q))\left(\operatorname{cf}\right.$. Lemma 5), we have that $\int_{\Omega} \operatorname{div}\left(\boldsymbol{u}-(\nabla q)_{I}\right) \operatorname{div} \boldsymbol{v}_{h}=0$ for all $\boldsymbol{v}_{h} \in \mathcal{V}_{h}$. Then,

$$
\begin{aligned}
\beta\left\|\boldsymbol{v}_{h}\right\|_{\mathrm{div}, \Omega}^{2} \leq\left(b_{h}\left(\boldsymbol{f}_{h}, \boldsymbol{v}_{h}\right)-\int_{\Omega} \boldsymbol{f}_{h} \cdot \boldsymbol{v}_{h}\right) & \\
& -\sum_{E \in \mathcal{T}_{h}}\left(b_{h}^{E}\left((\nabla q)_{I}-\boldsymbol{\Pi}_{h}^{E} \boldsymbol{u}, \boldsymbol{v}_{h}\right)+\int_{E}\left(\boldsymbol{\Pi}_{h}^{E} \boldsymbol{u}-\boldsymbol{u}\right) \cdot \boldsymbol{v}_{h}\right) .
\end{aligned}
$$

The first term on the right hand side can be bounded as follows:

$$
\begin{aligned}
b_{h}\left(\boldsymbol{f}_{h}, \boldsymbol{v}_{h}\right) & -\int_{\Omega} \boldsymbol{f}_{h} \cdot \boldsymbol{v}_{h}=\sum_{E \in \mathcal{T}_{h}}\left(b_{h}^{E}\left(\boldsymbol{f}_{h}, \boldsymbol{v}_{h}\right)-\int_{E} \boldsymbol{f}_{h} \cdot \boldsymbol{v}_{h}\right) \\
& =\sum_{E \in \mathcal{T}_{h}}\left(\int_{E} \boldsymbol{\Pi}_{h}^{E} \boldsymbol{f}_{h} \cdot \boldsymbol{\Pi}_{h}^{E} \boldsymbol{v}_{h}+S^{E}\left(\boldsymbol{f}_{h}-\boldsymbol{\Pi}_{h}^{E} \boldsymbol{f}_{h}, \boldsymbol{v}_{h}-\boldsymbol{\Pi} \boldsymbol{\Pi}_{h}^{E} \boldsymbol{v}_{h}\right)-\int_{E} \boldsymbol{f}_{h} \cdot \boldsymbol{v}_{h}\right) \\
& =\sum_{E \in \mathcal{T}_{h}} \int_{E}\left(\boldsymbol{\Pi}_{h}^{E} \boldsymbol{f}_{h}-\boldsymbol{f}_{h}\right) \cdot \boldsymbol{v}_{h}+\sum_{E \in \mathcal{T}_{h}} S^{E}\left(\boldsymbol{f}_{h}-\boldsymbol{\Pi}_{h}^{E} \boldsymbol{f}_{h}, \boldsymbol{v}_{h}-\boldsymbol{\Pi}_{h}^{E} \boldsymbol{v}_{h}\right),
\end{aligned}
$$

where we have used (3) to write the last equality. Now, from the symmetry of $S^{E}(\cdot, \cdot)$, (4), a Cauchy-Schwarz inequality and the fact that $\boldsymbol{\Pi}_{h}^{E}$ is an $\mathrm{L}^{2}(E)$-projection (cf. (3)), we have that

$$
\sum_{E \in \mathcal{T}_{h}} S^{E}\left(\boldsymbol{f}_{h}-\boldsymbol{\Pi}_{h}^{E} \boldsymbol{f}_{h}, \boldsymbol{v}_{h}-\boldsymbol{\Pi}_{h}^{E} \boldsymbol{v}_{h}\right) \leq \sum_{E \in \mathcal{T}_{h}} c_{1}\left\|\boldsymbol{f}_{h}-\boldsymbol{\Pi}_{h}^{E} \boldsymbol{f}_{h}\right\|_{0, E}\left\|\boldsymbol{v}_{h}\right\|_{0, E} .
$$

Therefore, using Cauchy-Schwarz inequality again,

$$
b_{h}\left(\boldsymbol{f}_{h}, \boldsymbol{v}_{h}\right)-\int_{\Omega} \boldsymbol{f}_{h} \cdot \boldsymbol{v}_{h} \leq C \sum_{E \in \mathcal{T}_{h}}\left\|\boldsymbol{f}_{h}-\boldsymbol{\Pi}_{h}^{E} \boldsymbol{f}_{h}\right\|_{0, E}\left\|\boldsymbol{v}_{h}\right\|_{0, E} .
$$

Substituting the above estimate in (14), from (7) and Cauchy-Schwarz inequality we obtain

$$
\begin{aligned}
\beta\left\|\boldsymbol{v}_{h}\right\|_{\mathrm{div}, \Omega}^{2} & \leq C \sum_{E \in \mathcal{T}_{h}}\left(\left\|\boldsymbol{f}_{h}-\boldsymbol{\Pi}_{h}^{E} \boldsymbol{f}_{h}\right\|_{0, E}+\left\|\boldsymbol{u}-(\nabla q)_{I}\right\|_{0, E}+\left\|\boldsymbol{u}-\boldsymbol{\Pi}_{h}^{E} \boldsymbol{u}\right\|_{0, E}\right)\left\|\boldsymbol{v}_{h}\right\|_{0, E} \\
& \leq C\left(\left\|\boldsymbol{f}_{h}-\boldsymbol{\Pi}_{h} \boldsymbol{f}_{h}\right\|_{0, \Omega}+\left\|\boldsymbol{u}-(\nabla q)_{I}\right\|_{0, \Omega}+\left\|\boldsymbol{u}-\boldsymbol{\Pi}_{h} \boldsymbol{u}\right\|_{0, \Omega}\right)\left\|\boldsymbol{v}_{h}\right\|_{\operatorname{div}, \Omega},
\end{aligned}
$$

with $\boldsymbol{\Pi}_{h}$ as defined in (12). Therefore, from (13),

$$
\left\|\left(\mathbf{T}-\mathbf{T}_{h}\right) \boldsymbol{f}_{h}\right\|_{\operatorname{div}, \Omega} \leq C\left(\left\|\boldsymbol{f}_{h}-\boldsymbol{\Pi}_{h} \boldsymbol{f}_{h}\right\|_{0, \Omega}+\left\|\boldsymbol{u}-\boldsymbol{\Pi}_{h} \boldsymbol{u}\right\|_{0, \Omega}+\left\|\boldsymbol{u}-(\nabla q)_{I}\right\|_{\operatorname{div}, \Omega}\right) .
$$

Thus, there only remains to estimate the three terms on the right-hand side above. For the first one we write $\boldsymbol{f}_{h}=\boldsymbol{\psi}+\nabla p$ with $\psi \in \mathcal{K}$ and $p \in \mathrm{H}^{1+s}(\Omega)$ as in Lemma 7 . Hence, by using this and Lemma 8 ,

$$
\begin{aligned}
\left\|\boldsymbol{f}_{h}-\boldsymbol{\Pi}_{h} \boldsymbol{f}_{h}\right\|_{0, \Omega} & \leq\left\|\boldsymbol{\psi}-\boldsymbol{\Pi}_{h} \boldsymbol{\psi}\right\|_{0, \Omega}+\left\|\nabla p-\boldsymbol{\Pi}_{h}(\nabla p)\right\|_{0, \Omega} \\
& \leq C\left(\|\boldsymbol{\psi}\|_{0, \Omega}+\left\|\nabla p-\boldsymbol{\Pi}_{h}(\nabla p)\right\|_{0, \Omega}\right) \\
& \leq C h^{s}\left\|\operatorname{div} \boldsymbol{f}_{h}\right\|_{0, \Omega} .
\end{aligned}
$$


On the other hand, we have that $\boldsymbol{u}=\mathbf{T}(\boldsymbol{\psi}+\nabla p)=\boldsymbol{\psi}+\mathbf{T}(\nabla p)$ and, from Lemmas 3 and $2, \mathbf{T}(\nabla p)=\nabla q$ and $\boldsymbol{\psi}=\boldsymbol{\varphi}$. Moreover, by virtue of Theorem $1, q \in \mathrm{H}^{1+s}(\Omega)$ and

$$
\|\nabla q\|_{s, \Omega} \leq C\|\nabla p\|_{\operatorname{div}, \Omega} \leq C\left\|\boldsymbol{f}_{h}\right\|_{\operatorname{div}, \Omega},
$$

whereas estimate (11) still holds true for $\boldsymbol{\psi}$ :

$$
\|\boldsymbol{\psi}\|_{0, \Omega} \leq C h^{s}\left\|\operatorname{div} \boldsymbol{f}_{h}\right\|_{0, \Omega} .
$$

Then, using that $\boldsymbol{\Pi}_{h}$ is an $\left[\mathrm{L}^{2}(\Omega)\right]^{2}$-projection, from Lemmas 7 and 8 we have

$$
\begin{aligned}
\left\|\boldsymbol{u}-\boldsymbol{\Pi}_{h} \boldsymbol{u}\right\|_{0, \Omega} & \left.\leq\left\|\boldsymbol{\psi}-\boldsymbol{\Pi}_{h} \boldsymbol{\psi}\right\|_{0, \Omega}+\left\|\nabla q-\boldsymbol{\Pi}_{h}(\nabla q)\right\|_{0, \Omega}\right) \\
& \leq C\left(\|\boldsymbol{\psi}\|_{0, \Omega}+\left\|\nabla q-\boldsymbol{\Pi}_{h}(\nabla q)\right\|_{0, \Omega}\right) \\
& \leq C h^{s}\left\|\operatorname{div} \boldsymbol{f}_{h}\right\|_{0, \Omega}+C h^{s}\|\nabla q\|_{s, \Omega} \\
& \leq C h^{s}\left\|\boldsymbol{f}_{h}\right\|_{\operatorname{div}, \Omega} .
\end{aligned}
$$

Finally, using once more that $\boldsymbol{u}=\boldsymbol{\psi}+\nabla q$ and Lemmas 7,6 and 5, we write

$$
\begin{aligned}
\left\|\boldsymbol{u}-(\nabla q)_{I}\right\|_{\operatorname{div}, \Omega} \leq\|\boldsymbol{\psi}\|_{\operatorname{div}, \Omega}+\left\|\nabla q-(\nabla q)_{I}\right\|_{\operatorname{div}, \Omega} \\
\quad \leq C h^{s}\left\|\operatorname{div} \boldsymbol{f}_{h}\right\|_{0, \Omega}+\left\|\nabla q-(\nabla q)_{I}\right\|_{0, \Omega}+\left\|\operatorname{div}(\nabla q)-\operatorname{div}\left((\nabla q)_{I}\right)\right\|_{0, \Omega} \\
\leq C h^{s}\left\|\boldsymbol{f}_{h}\right\|_{\operatorname{div}, \Omega}+C\left(h^{s}|\nabla q|_{s, \Omega}+h\|\operatorname{div}(\nabla q)\|_{0, \Omega}\right)+C h|\operatorname{div}(\nabla q)|_{1, \Omega} \\
\leq C h^{s}\left\|\boldsymbol{f}_{h}\right\|_{\operatorname{div}, \Omega},
\end{aligned}
$$

where, we have used that $\nabla q=\mathbf{T}(\nabla p)$ and, hence, since $\nabla p \in \mathcal{G} \cap \mathcal{V}$, from Theorem 1 $\operatorname{div}(\nabla q) \in \mathrm{H}^{1}(\Omega)$ and $\|\operatorname{div}(\nabla q)\|_{1, \Omega} \leq C\|\nabla p\|_{\operatorname{div}, \Omega} \leq C\left\|\boldsymbol{f}_{h}\right\|_{\operatorname{div}, \Omega}$.

Collecting the previous estimates, we obtain

$$
\left\|\left(\mathbf{T}-\mathbf{T}_{h}\right) \boldsymbol{f}_{h}\right\|_{\operatorname{div}, \Omega} \leq C h^{s}\left\|\boldsymbol{f}_{h}\right\|_{\operatorname{div}, \Omega}
$$

and we end the proof.

Now, we are in a position to conclude property $\mathbf{P} 1$.

Corollary 1 There exists $C>0$, independent of $h$, such that

$$
\left\|\mathbf{T}-\mathbf{T}_{h}\right\|_{h} \leq C h^{s} .
$$

Proof Given $\boldsymbol{v}_{h} \in \mathcal{V}_{h}$, we have that $\boldsymbol{v}_{h}=\boldsymbol{\psi}_{h}+\boldsymbol{f}_{h}$ with $\boldsymbol{\psi}_{h} \in \mathcal{K}_{h}$ and $\boldsymbol{f}_{h} \in \mathcal{K}_{h}^{\perp}$, then

$$
\left\|\left(\mathbf{T}-\mathbf{T}_{h}\right) \boldsymbol{v}_{h}\right\|_{\operatorname{div}, \Omega}=\left\|\left(\mathbf{T}-\mathbf{T}_{h}\right) \boldsymbol{f}_{h}\right\|_{\operatorname{div}, \Omega} \leq C h^{s}\left\|\boldsymbol{f}_{h}\right\|_{\operatorname{div}, \Omega},
$$

where the last inequality follows from Lemma 9 . The proof follows by noting that, since $\boldsymbol{v}_{h}=\boldsymbol{\psi}_{h}+\boldsymbol{f}_{h}$ is an orthogonal decomposition in $\mathrm{H}(\operatorname{div} ; \Omega)$, we have that $\left\|\boldsymbol{f}_{h}\right\|_{\text {div }, \Omega} \leq$ $\left\|\boldsymbol{v}_{h}\right\|_{\operatorname{div}, \Omega}$.

In order to establish spectral convergence and error estimates, we recall some other basic definitions from spectral theory.

Given a generic linear bounded operator $\mathbf{S}: \mathcal{V} \longrightarrow \mathcal{V}$ defined on a Hilbert space $\mathcal{V}$, the spectrum of $\mathbf{S}$ is the set $\operatorname{sp}(\mathbf{S}):=\{z \in \mathbb{C}:(z \mathbf{I}-\mathbf{S})$ is not invertible $\}$ and the resolvent set of $\mathbf{S}$ is its complement $\rho(\mathbf{S}):=\mathbb{C} \backslash \operatorname{sp}(\mathbf{S})$. For any $z \in \rho(\mathbf{S}), R_{z}(\mathbf{S}):=$ $(z \mathbf{I}-\mathbf{S})^{-1}: \mathcal{V} \longrightarrow \mathcal{V}$ is the resolvent operator of $\mathbf{S}$ corresponding to $z$.

The following two results are consequence of property P1, see [24, Lemma 1 and Theorem 1]. 
Lemma 10 Let us assume that $\mathbf{P} 1$ holds true and let $F \subset \rho(\mathbf{T})$ be closed. Then, there exist positive constants $C$ and $h_{0}$ independent of $h$, such that for $h<h_{0}$

$$
\sup _{\boldsymbol{v}_{h} \in \mathcal{V}_{h}}\left\|R_{z}\left(\mathbf{T}_{h}\right) \boldsymbol{v}_{h}\right\|_{\operatorname{div}, \Omega} \leq C\left\|\boldsymbol{v}_{h}\right\|_{\operatorname{div}, \Omega} \quad \forall z \in F
$$

Theorem 4 Let $U \subset \mathbb{C}$ be an open set containing $\operatorname{sp}(\mathbf{T})$. Then, there exists $h_{0}>0$ such that $\operatorname{sp}\left(\mathbf{T}_{h}\right) \subset U$ for all $h<h_{0}$.

An immediate consequence of this theorem and Corollary 1 is that the proposed virtual element method does not introduce spurious modes with eigenvalues interspersed among those with a physical meaning. Let us remark that such a spectral pollution could be in principle expected from the fact that the corresponding solution operator $\mathbf{T}$ has an infinite-dimensional eigenvalue $\mu=1$ (see $[12,16,17]$ ).

By applying the results from [24, Section 2] to our problem, we conclude the spectral convergence of $\mathbf{T}_{h}$ to $\mathbf{T}$ as $h \rightarrow 0$. More precisely, let $\mu \in(0,1)$ be an isolated eigenvalue of $\mathbf{T}$ with multiplicity $m$ and let $\mathcal{C}$ be an open circle in the complex plane centered at $\mu$, such that $\mu$ is the only eigenvalue of $\mathbf{T}$ lying in $\mathcal{C}$ and $\partial \mathcal{C} \cap \operatorname{sp}(\mathbf{T})=\emptyset$. Then, according to $\left[24\right.$, Section 2], for $h$ small enough there exist $m$ eigenvalues $\mu_{h}^{(1)}, \ldots, \mu_{h}^{(m)}$ of $\mathbf{T}_{h}$ (repeated according to their respective multiplicities) which lie in $\mathcal{C}$. Therefore, these eigenvalues $\mu_{h}^{(1)}, \ldots, \mu_{h}^{(m)}$ converge to $\mu$ as $h$ goes to zero.

Our next step is to obtain error estimates for the spectral approximation. The classical reference for this issue on non-compact operators is [25]. However, we cannot apply the results from this reference directly to our problem, because of the variational crimes in the bilinear forms used to define the operator $\mathbf{T}_{h}$. Therefore, we need to extend the results from this reference to our case. With this purpose, we follow an approach inspired by those of $[13,31]$.

Consider the eigenspace $\mathcal{E}$ of $\mathbf{T}$ corresponding to $\mu$ and the $\mathbf{T}_{h}$-invariant subspace $\mathcal{E}_{h}$ spanned by the eigenspaces of $\mathbf{T}_{h}$ corresponding to $\mu_{h}^{(1)}, \ldots, \mu_{h}^{(m)}$. As a consequence of Lemma 10, we have for $h$ small enough

$$
\left\|\left(z \mathbf{I}-\mathbf{T}_{h}\right) \boldsymbol{v}_{h}\right\|_{\operatorname{div}, \Omega} \geq C\left\|\boldsymbol{v}_{h}\right\|_{\operatorname{div}, \Omega} \quad \forall \boldsymbol{v}_{h} \in \mathcal{V}_{h}, \quad \forall z \in \partial \mathcal{C}
$$

Let $\mathbf{P}_{h}: \mathcal{V} \longrightarrow \mathcal{V}_{h} \hookrightarrow \mathcal{V}$ be the projector with range $\mathcal{V}_{h}$ defined by the relation

$$
a\left(\mathbf{P}_{h} \boldsymbol{u}-\boldsymbol{u}, \boldsymbol{v}_{h}\right)=0 \quad \forall \boldsymbol{v}_{h} \in \mathcal{V}_{h}
$$

In our case, the bilinear form $a(\cdot, \cdot)$ is the inner product of $\mathcal{V}$, so that $\left\|\mathbf{P}_{h} \boldsymbol{u}\right\|_{\text {div }, \Omega} \leq$ $\|\boldsymbol{u}\|_{\operatorname{div}, \Omega}$ and

$$
\left\|\boldsymbol{u}-\mathbf{P}_{h} \boldsymbol{u}\right\|_{\operatorname{div}, \Omega}=\delta\left(\boldsymbol{u}, \mathcal{V}_{h}\right) \quad \forall \boldsymbol{u} \in \mathcal{V}
$$

Now, we define $\widehat{\mathbf{T}}_{h}:=\mathbf{T}_{h} \mathbf{P}_{h}: \mathcal{V} \longrightarrow \mathcal{V}_{h}$. Notice that $\operatorname{sp}\left(\widehat{\mathbf{T}}_{h}\right)=\operatorname{sp}\left(\mathbf{T}_{h}\right) \cup\{0\}$. Furthermore, we have the following result (cf. [25, Lemma 1]).

Lemma 11 There exist $h_{0}>0$ and $C>0$ such that

$$
\left\|R_{z}\left(\widehat{\mathbf{T}}_{h}\right)\right\|_{\operatorname{div}, \Omega} \leq C \quad \forall z \in \partial \mathcal{C}, \quad \forall h \leq h_{0}
$$


Proof Since $\widehat{\mathbf{T}}_{h}$ is compact, it suffices to check that $\left\|\left(z \mathbf{I}-\widehat{\mathbf{T}}_{h}\right) \boldsymbol{v}\right\|_{\operatorname{div}, \Omega} \geq C\|\boldsymbol{v}\|_{\operatorname{div}, \Omega}$ $\forall \boldsymbol{v} \in \mathcal{V}$ and $\forall z \in \partial \mathcal{C}$. By using (16) and basic properties of the projector $\mathbf{P}_{h}$, we obtain

$$
\begin{aligned}
\|\boldsymbol{v}\|_{\operatorname{div}, \Omega} & \leq\left\|\mathbf{P}_{h} \boldsymbol{v}\right\|_{\operatorname{div}, \Omega}+\left\|\boldsymbol{v}-\mathbf{P}_{h} \boldsymbol{v}\right\|_{\operatorname{div}, \Omega} \\
& \leq C\left\|\left(z \mathbf{I}-\mathbf{T}_{h}\right) \mathbf{P}_{h} \boldsymbol{v}\right\|_{\operatorname{div}, \Omega}+|z|^{-1}\left\|z\left(\boldsymbol{v}-\mathbf{P}_{h} \boldsymbol{v}\right)\right\|_{\operatorname{div}, \Omega} \\
& \leq C\left\|\left(z \mathbf{I}-\widehat{\mathbf{T}}_{h}\right) \mathbf{P}_{h} \boldsymbol{v}\right\|_{\operatorname{div}, \Omega}+|z|^{-1}\left\|z\left(\boldsymbol{v}-\mathbf{P}_{h} \boldsymbol{v}\right)-\widehat{\mathbf{T}}_{h}\left(\boldsymbol{v}-\mathbf{P}_{h} \boldsymbol{v}\right)\right\|_{\operatorname{div}, \Omega} \\
& \left.=C\left\|\mathbf{P}_{h}\left(z \mathbf{I}-\widehat{\mathbf{T}}_{h}\right) \boldsymbol{v}\right\|_{\operatorname{div}, \Omega}+|z|^{-1} \|\left(\mathbf{I}-\mathbf{P}_{h}\right)\left(z \mathbf{I}-\widehat{\mathbf{T}}_{h}\right) \boldsymbol{v}\right) \|_{\operatorname{div}, \Omega} \\
& \leq C\left\|\left(z \mathbf{I}-\widehat{\mathbf{T}}_{h}\right) \boldsymbol{v}\right\|_{\operatorname{div}, \Omega},
\end{aligned}
$$

where we have used that the curve $\partial \mathcal{C}$ is bounded away from 0.

Next, we introduce the following spectral projectors (the second one, is well defined at least for $h$ small enough):

- the spectral projector of $\mathbf{T}$ relative to $\mu: \quad \mathbf{F}:=\frac{1}{2 \pi i} \int_{\partial \mathcal{C}} R_{z}(\mathbf{T}) d z ;$

- the spectral projector of $\widehat{\mathbf{T}}_{h}$ relative to $\mu_{h}^{(1)}, \ldots, \mu_{h}^{(m)}: \quad \widehat{\mathbf{F}}_{h}:=\frac{1}{2 \pi i} \int_{\partial \mathcal{C}} R_{z}\left(\widehat{\mathbf{T}}_{h}\right) d z$.

We also introduce the quantities

$$
\gamma_{h}:=\delta\left(\mathcal{E}, \mathcal{V}_{h}\right) \quad \text { and } \quad \eta_{h}:=\sup _{\boldsymbol{w} \in \mathcal{E}} \frac{\left\|\boldsymbol{w}-\boldsymbol{\Pi}_{h} \boldsymbol{w}\right\|_{0, \Omega}}{\|\boldsymbol{w}\|_{\operatorname{div}}, \Omega}
$$

These two quantities are bounded as follows:

$$
\gamma_{h} \leq C h^{\min \{\tilde{s}, k+1\}} \quad \text { and } \quad \eta_{h} \leq C h^{\min \{\tilde{s}, k+1\}},
$$

where $\tilde{s}>1 / 2$ is such that $\mathcal{E} \subset\left[\mathrm{H}^{\tilde{s}}(\Omega)\right]^{2}$ (cf. Theorem 2). In fact, the first estimate follows from Lemmas 5 and 6 and Theorem 2(ii), whereas the latter follows from the fact that $\mathcal{E} \subset \mathcal{G} \cap \mathcal{V}$, Lemma 8 and Theorem 2(ii) again.

The following estimate is a variation of Lemma 3 from [25]) that will be used to prove convergence of the eigenspaces.

Lemma 12 There exist positive constants $h_{0}$ and $C$ such that, for all $h<h_{0}$,

$$
\left\|\left.\left(\mathbf{F}-\widehat{\mathbf{F}}_{h}\right)\right|_{\mathcal{E}}\right\|_{\operatorname{div}, \Omega} \leq C\left\|\left(\mathbf{T}-\widehat{\mathbf{T}}_{h}\right) \mid \mathcal{E}\right\|_{\operatorname{div}, \Omega} \leq C\left(\gamma_{h}+\eta_{h}\right) .
$$

Proof The first inequality is proved using the same arguments of [25, Lemma 3] and Lemma 11. For the other estimate, let $\boldsymbol{f} \in \mathcal{E}, \boldsymbol{w}:=\mathbf{T} \boldsymbol{f}$ and $\boldsymbol{w}_{h}:=\widehat{\mathbf{T}}_{h} \boldsymbol{f}=\mathbf{T}_{h} \mathbf{P}_{h} \boldsymbol{f}$. Note that, by Theorem 2(ii), $\boldsymbol{f} \in \nabla\left(\mathrm{H}^{1+\tilde{s}}(\Omega)\right), \tilde{s}>1 / 2$. By using the first Strang lemma (see, for instance, [23, Theorem 4.1.1]), we have

$$
\begin{aligned}
\left\|\boldsymbol{w}-\boldsymbol{w}_{h}\right\|_{\operatorname{div}, \Omega} \leq C( & \left\|\boldsymbol{w}-\mathbf{P}_{h} \boldsymbol{w}\right\|_{\operatorname{div}, \Omega}+\sup _{\boldsymbol{v}_{h} \in \mathcal{V}_{h}} \frac{\left|b\left(\mathbf{P}_{h} \boldsymbol{w}, \boldsymbol{v}_{h}\right)-b_{h}\left(\mathbf{P}_{h} \boldsymbol{w}, \boldsymbol{v}_{h}\right)\right|}{\left\|\boldsymbol{v}_{h}\right\|_{\operatorname{div}, \Omega}} \\
& \left.+\sup _{\boldsymbol{v}_{h} \in \mathcal{V}_{h}} \frac{\left|b\left(\boldsymbol{f}, \boldsymbol{v}_{h}\right)-b_{h}\left(\mathbf{P}_{h} \boldsymbol{f}, \boldsymbol{v}_{h}\right)\right|}{\left\|\boldsymbol{v}_{h}\right\|_{\operatorname{div}, \Omega},}\right)
\end{aligned}
$$


and by proceeding as in the proof of Lemma 9 to derive (15), we obtain

$$
\begin{aligned}
\left|b\left(\mathbf{P}_{h} \boldsymbol{w}, \boldsymbol{v}_{h}\right)-b_{h}\left(\mathbf{P}_{h} \boldsymbol{w}, \boldsymbol{v}_{h}\right)\right| & \leq C \sum_{E \in \mathcal{T}_{h}}\left\|\mathbf{P}_{h} \boldsymbol{w}-\boldsymbol{\Pi}_{h}^{E} \mathbf{P}_{h} \boldsymbol{w}\right\|_{0, E}\left\|\boldsymbol{v}_{h}\right\|_{0, E} \\
& \leq C \sum_{E \in \mathcal{T}_{h}}\left\|\left(\mathbf{I}-\boldsymbol{\Pi}_{h}^{E}\right)\left(\mathbf{P}_{h} \boldsymbol{w}-\boldsymbol{w}\right)+\left(\mathbf{I}-\boldsymbol{\Pi}_{h}^{E}\right) \boldsymbol{w}\right\|_{0, E} \\
& \leq C\left(\left\|\boldsymbol{w}-\mathbf{P}_{h} \boldsymbol{w}\right\|_{0, \Omega}+\left\|\boldsymbol{w}-\boldsymbol{\Pi}_{h} \boldsymbol{w}\right\|_{0, \Omega}\right)\left\|\boldsymbol{v}_{h}\right\|_{\mathrm{div}, \Omega} .
\end{aligned}
$$

On the other hand,

$$
\begin{aligned}
\left|b\left(\boldsymbol{f}, \boldsymbol{v}_{h}\right)-b_{h}\left(\mathbf{P}_{h} \boldsymbol{f}, \boldsymbol{v}_{h}\right)\right| & \leq\left|b\left(\boldsymbol{f}-\mathbf{P}_{h} \boldsymbol{f}, \boldsymbol{v}_{h}\right)\right|+\left|b\left(\mathbf{P}_{h} \boldsymbol{f}, \boldsymbol{v}_{h}\right)-b_{h}\left(\mathbf{P}_{h} \boldsymbol{f}, \boldsymbol{v}_{h}\right)\right| \\
& \leq C\left(\left\|\boldsymbol{f}-\mathbf{P}_{h} \boldsymbol{f}\right\|_{0, \Omega}\left\|\boldsymbol{v}_{h}\right\|_{0, \Omega}\right)+\left|b\left(\mathbf{P}_{h} \boldsymbol{f}, \boldsymbol{v}_{h}\right)-b_{h}\left(\mathbf{P}_{h} \boldsymbol{f}, \boldsymbol{v}_{h}\right)\right| \\
& \leq C\left(\left\|\boldsymbol{f}-\mathbf{P}_{h} \boldsymbol{f}\right\|_{0, \Omega}+\left\|\boldsymbol{f}-\boldsymbol{\Pi}_{h} \boldsymbol{f}\right\|_{0, \Omega}\right)\left\|\boldsymbol{v}_{h}\right\|_{\mathrm{div}, \Omega},
\end{aligned}
$$

where, for the last inequality, we have used the same argument as above. Then, we have

$$
\begin{aligned}
\left\|\boldsymbol{w}-\boldsymbol{w}_{h}\right\|_{\mathrm{div}, \Omega} \leq & C\left(\left\|\boldsymbol{w}-\mathbf{P}_{h} \boldsymbol{w}\right\|_{\mathrm{div}, \Omega}+\left\|\boldsymbol{w}-\boldsymbol{\Pi}_{h} \boldsymbol{w}\right\|_{0, \Omega}\right. \\
& \left.\quad+\left\|\boldsymbol{f}-\mathbf{P}_{h} \boldsymbol{f}\right\|_{0, \Omega}+\left\|\boldsymbol{f}-\boldsymbol{\Pi}_{h} \boldsymbol{f}\right\|_{0, \Omega}\right) \\
\leq & C\left(\gamma_{h}+\left\|\boldsymbol{w}-\boldsymbol{\Pi}_{h} \boldsymbol{w}\right\|_{0, \Omega}+\left\|\boldsymbol{f}-\boldsymbol{\Pi}_{h} \boldsymbol{f}\right\|_{0, \Omega}\right) \\
= & C\left(\gamma_{h}+\left(1+\mu^{-1}\right)\left\|\boldsymbol{w}-\boldsymbol{\Pi}_{h} \boldsymbol{w}\right\|_{0, \Omega}\right) \\
\leq & C\left(\gamma_{h}+\eta_{h}\right),
\end{aligned}
$$

where we have used that, for $\boldsymbol{f} \in \mathcal{E}, \boldsymbol{w}:=\mathbf{T} \boldsymbol{f}=\mu \boldsymbol{f}$. Thus, we conclude the proof.

To prove an error estimate for the eigenspaces, we also need the following result.

Lemma 13 Let

$$
\boldsymbol{\Lambda}_{h}:=\left.\widehat{\mathbf{F}}_{h}\right|_{\mathcal{E}}: \mathcal{E} \longrightarrow \mathcal{E}_{h}
$$

For $h$ small enough, the operator $\boldsymbol{\Lambda}_{h}$ is invertible and there exists $C$ independent of $h$ such that

$$
\left\|\boldsymbol{\Lambda}_{h}^{-1}\right\| \leq C
$$

Proof It follows by proceeding as in the proof of Lemma 2 from [25], by using Lemma 12 and the fact that $\gamma_{h} \rightarrow 0$ and $\eta_{h} \rightarrow 0$ as $h \rightarrow 0$ (cf. (17)).

The following theorem shows that the eigenspace of $\mathbf{T}_{h}$ (which coincides with that of $\widehat{\mathbf{T}}_{h}$ ) approximates the eigenspace of $\mathbf{T}$.

Theorem 5 There exists $C>0$ such that,

$$
\widehat{\delta}\left(\mathcal{E}, \mathcal{E}_{h}\right) \leq C\left(\gamma_{h}+\eta_{h}\right) .
$$

Proof It follows by arguing exactly as in the proof of Theorem 1 from [25] and using Lemmas 12 and 13. 
Finally, we will prove a double-order error estimate for the eigenvalues. With this aim, let $\lambda:=\frac{1}{\mu}-1$ be the eigenvalue of Problem 1 with eigenspace $\mathcal{E}$. Let $\lambda_{h}^{i}:=\frac{1}{\mu_{h}^{i}}-1$, $i=1, \ldots, m$, be the eigenvalues of Problem 3 with invariant subspace $\mathcal{E}_{h}$. We have the following result.

Theorem 6 There exist positive constants $C$ and $h_{0}$ independent of $h$, such that, for $h<$ $h_{0}$,

$$
\left|\lambda-\lambda_{h}^{(i)}\right| \leq C\left(\gamma_{h}^{2}+\eta_{h}^{2}\right), \quad i=1, \ldots, m
$$

Proof Let $\boldsymbol{w}_{h} \in \mathcal{E}_{h}$ be an eigenfunction corresponding to one of the eigenvalues $\lambda_{h}^{(i)}$ $(i=1, \ldots, m)$ with $\left\|\boldsymbol{w}_{h}\right\|_{\operatorname{div}, \Omega}=1$. According to Theorem $5, \delta\left(\boldsymbol{w}_{h}, \mathcal{E}\right) \leq C\left(\gamma_{h}+\eta_{h}\right)$. It follows that there exists $\boldsymbol{w} \in \mathcal{E}$ such that

$$
\left\|\boldsymbol{w}-\boldsymbol{w}_{h}\right\|_{\text {div }, \Omega} \leq C\left(\gamma_{h}+\eta_{h}\right) .
$$

Moreover, it is easy to check that $\boldsymbol{w}$ can be chosen normalized in $\mathrm{H}(\operatorname{div} ; \Omega)$-norm.

From the symmetry of the bilinear forms and the facts that $\boldsymbol{w}$ and $\boldsymbol{w}_{h}$ are solutions of Problem 1 and 3, respectively, we have

$$
\begin{aligned}
\int_{\Omega} \operatorname{div}\left(\boldsymbol{w}-\boldsymbol{w}_{h}\right)^{2}-\lambda \int_{\Omega} & \left(\boldsymbol{w}-\boldsymbol{w}_{h}\right)^{2}=\lambda_{h}^{(i)} b_{h}\left(\boldsymbol{w}_{h}, \boldsymbol{w}_{h}\right)-\lambda b\left(\boldsymbol{w}_{h}, \boldsymbol{w}_{h}\right) \\
& =\lambda_{h}^{(i)}\left(b_{h}\left(\boldsymbol{w}_{h}, \boldsymbol{w}_{h}\right)-b\left(\boldsymbol{w}_{h}, \boldsymbol{w}_{h}\right)\right)+\left(\lambda_{h}^{(i)}-\lambda\right) b\left(\boldsymbol{w}_{h}, \boldsymbol{w}_{h}\right),
\end{aligned}
$$

from which we obtain the following identity:

$$
\begin{aligned}
\left(\lambda_{h}^{(i)}-\lambda\right) b\left(\boldsymbol{w}_{h}, \boldsymbol{w}_{h}\right)= & \int_{\Omega} \operatorname{div}\left(\boldsymbol{w}-\boldsymbol{w}_{h}\right)^{2}-\lambda \int_{\Omega}\left(\boldsymbol{w}-\boldsymbol{w}_{h}\right)^{2} \\
& -\lambda_{h}^{(i)}\left(b_{h}\left(\boldsymbol{w}_{h}, \boldsymbol{w}_{h}\right)-b\left(\boldsymbol{w}_{h}, \boldsymbol{w}_{h}\right)\right) .
\end{aligned}
$$

The next step is to estimate each term on the right hand side above. The first and the second ones are easily bounded by using the Cauchy-Schwarz inequality and (18):

$$
\left|\int_{\Omega} \operatorname{div}\left(\boldsymbol{w}-\boldsymbol{w}_{h}\right)^{2}-\lambda \int_{\Omega}\left(\boldsymbol{w}-\boldsymbol{w}_{h}\right)^{2}\right| \leq C\left\|\boldsymbol{w}-\boldsymbol{w}_{h}\right\|_{\mathrm{div}, \Omega}^{2} \leq C\left(\gamma_{h}^{2}+\eta_{h}^{2}\right) .
$$

For the third term, we use (4)-(5) to write

$$
\begin{aligned}
\left|b_{h}\left(\boldsymbol{w}_{h}, \boldsymbol{w}_{h}\right)-b\left(\boldsymbol{w}_{h}, \boldsymbol{w}_{h}\right)\right| & \\
& =\left|\sum_{E \in \mathcal{T}_{h}}\left(\int_{E}\left(\boldsymbol{\Pi}_{h}^{E} \boldsymbol{w}_{h}\right)^{2}+S^{E}\left(\boldsymbol{w}_{h}-\boldsymbol{\Pi}_{h}^{E} \boldsymbol{w}_{h}, \boldsymbol{w}_{h}-\boldsymbol{\Pi}_{h}^{E} \boldsymbol{w}_{h}\right)\right)-\sum_{E \in \mathcal{T}_{h}} \int_{E}\left(\boldsymbol{w}_{h}\right)^{2}\right| \\
& \leq\left|\sum_{E \in \mathcal{T}_{h}}\left(\left\|\boldsymbol{\Pi}_{h}^{E} \boldsymbol{w}_{h}\right\|_{0, E}^{2}-\left\|\boldsymbol{w}_{h}\right\|_{0, E}^{2}\right)\right|+\sum_{E \in \mathcal{T}_{h}} c_{1} \int_{E}\left(\boldsymbol{w}_{h}-\boldsymbol{\Pi}_{h}^{E} \boldsymbol{w}_{h}\right)^{2} \\
& =\sum_{E \in \mathcal{T}_{h}}\left\|\boldsymbol{w}_{h}-\boldsymbol{\Pi}_{h}^{E} \boldsymbol{w}_{h}\right\|_{0, E}^{2}+c_{1} \sum_{E \in \mathcal{T}_{h}}\left\|\boldsymbol{w}_{h}-\boldsymbol{\Pi}_{h}^{E} \boldsymbol{w}_{h}\right\|_{0, E}^{2} \\
& \leq C\left\|\boldsymbol{w}_{h}-\boldsymbol{\Pi}_{h} \boldsymbol{w}_{h}\right\|_{0, \Omega}^{2} \\
& \leq C\left(\left\|\boldsymbol{w}_{h}-\boldsymbol{w}\right\|_{0, \Omega}^{2}+\left\|\boldsymbol{w}-\boldsymbol{\Pi}_{h} \boldsymbol{w}\right\|_{0, \Omega}^{2}+\left\|\boldsymbol{\Pi}_{h}\left(\boldsymbol{w}-\boldsymbol{w}_{h}\right)\right\|_{0, \Omega}^{2}\right) .
\end{aligned}
$$


Then, from the last inequality, the definition of $\eta_{h}$, the fact that $\boldsymbol{\Pi}_{h}$ is an $\left[\mathrm{L}^{2}(\Omega)\right]^{2}-$ projection and (18), we obtain

$$
\left|b\left(\boldsymbol{w}_{h}, \boldsymbol{w}_{h}\right)-b_{h}\left(\boldsymbol{w}_{h}, \boldsymbol{w}_{h}\right)\right| \leq C\left(\gamma_{h}^{2}+\eta_{h}^{2}\right) .
$$

On the other hand, from the stability property (7),

$$
\left\|\operatorname{div} \boldsymbol{w}_{h}\right\|_{0, \Omega}^{2}=\lambda_{h}^{(i)} b_{h}\left(\boldsymbol{w}_{h}, \boldsymbol{w}_{h}\right) \leq \lambda_{h}^{(i)} \alpha^{*}\left\|\boldsymbol{w}_{h}\right\|_{0, \Omega}^{2},
$$

hence

$$
\left(1+\lambda_{h}^{(i)} \alpha^{*}\right)\left\|\boldsymbol{w}_{h}\right\|_{0, \Omega}^{2} \geq\left\|\boldsymbol{w}_{h}\right\|_{\operatorname{div}, \Omega}^{2}=1 .
$$

Therefore, since $\lambda_{h}^{(i)} \rightarrow \lambda$ as $h$ goes to zero, the theorem follows from (19), (20), (21) and the inequality above.

As shown in Theorem 2(ii), the eigenfunctions satisfy additional regularity. The following result shows that this implies an optimal order of convergence for the numerical method.

Corollary 2 If $\mathcal{E} \subset\left[\mathrm{H}^{\tilde{s}}(\Omega)\right]^{2}$ with $\tilde{s}>1 / 2$, then

$$
\widehat{\delta}\left(\mathcal{E}, \mathcal{E}_{h}\right) \leq C h^{\min \{\tilde{s}, k+1\}} .
$$

and

$$
\left|\lambda-\lambda_{h}^{(i)}\right| \leq C h^{2 \min \{\tilde{s}, k+1\}}, \quad i=1, \ldots, m .
$$

Proof It follows from the above theorems and the estimates (17).

\section{Numerical results}

Following the ideas proposed in [7], we have implemented in a MATLAB code a lowestorder VEM $(k=0)$ on arbitrary polygonal meshes. We report in this section a couple of numerical tests which allowed us to assess the theoretical results proved above.

To complete the choice of the VEM, we had to fix the bilinear form $S^{E}(\cdot, \cdot)$ satisfying (4) to be used. To do this, we proceeded as in [6]. For each element $E \in \mathcal{T}_{h}$ with edges $e_{1}, \ldots, e_{N_{E}}$, let $\left\{\boldsymbol{\varphi}_{1}, \ldots, \boldsymbol{\varphi}_{N_{E}}\right\}$ be the dual basis of $\mathcal{V}_{h}^{E}$ associated with the degrees of freedom (1); namely, $\boldsymbol{\varphi}_{i} \in \mathcal{V}_{h}^{E}$ are such that

$$
\int_{e_{j}} \boldsymbol{\varphi}_{i} \cdot \boldsymbol{n} d s=\delta_{i j}, \quad i, j=1, \ldots, N_{E}
$$

Therefore, $\left\|\boldsymbol{\varphi}_{i}\right\|_{\infty, E} \simeq \frac{1}{h_{E}}$, namely, there exists $C>0$ such that

$$
\frac{1}{C h_{E}} \leq\left\|\boldsymbol{\varphi}_{i}\right\|_{\infty, E} \leq \frac{C}{h_{E}}, \quad i=1, \ldots, N_{E}
$$

Hence, a natural choice for $S^{E}(\cdot, \cdot)$ is given by

$$
S^{E}\left(\boldsymbol{u}_{h}, \boldsymbol{v}_{h}\right):=\sigma_{E} \sum_{k=1}^{N_{E}}\left(\int_{e_{k}} \boldsymbol{u}_{h} \cdot \boldsymbol{n}\right)\left(\int_{e_{k}} \boldsymbol{v}_{h} \cdot \boldsymbol{n}\right), \quad \boldsymbol{u}_{h}, \boldsymbol{v}_{h} \in \mathcal{V}_{h}^{E},
$$

where $\sigma_{E}$ is the so-called stability constant which will be taken of the order of unity (see for instance $[6]$ ). 
5.1 Test 1: Rectangular acoustic cavity

In this test, the domain is a rectangle $\Omega:=(0, a) \times(0, b)$, in which case the exact analytic solution is known. The non vanishing eigenvalues of Problem 1 are given by

$$
\lambda_{n m}:=\pi^{2}\left(\left(\frac{n}{a}\right)^{2}+\left(\frac{m}{b}\right)^{2}\right), \quad n, m=0,1,2, \ldots, \quad n+m \neq 0
$$

while the corresponding eigenfunctions are

$$
\boldsymbol{w}_{n m}:=\left(\begin{array}{l}
\frac{n}{a} \sin \frac{n \pi x}{a} \cos \frac{m \pi y}{b} \\
\frac{m}{b} \cos \frac{n \pi x}{a} \sin \frac{m \pi y}{b}
\end{array}\right) .
$$

We have used $a=1$ and $b=1.1$. The stability constant has been taken $\sigma_{E}=1$. We have used three different families of meshes (see Figure 1):

$-\mathcal{T}_{h}^{1}:$ triangular meshes;

$-\mathcal{T}_{h}^{2}$ : rectangular meshes;

$-\mathcal{T}_{h}^{3}$ : hexagonal meshes.

The refinement parameter $N$ used to label each mesh is the number of elements intersecting each edge.
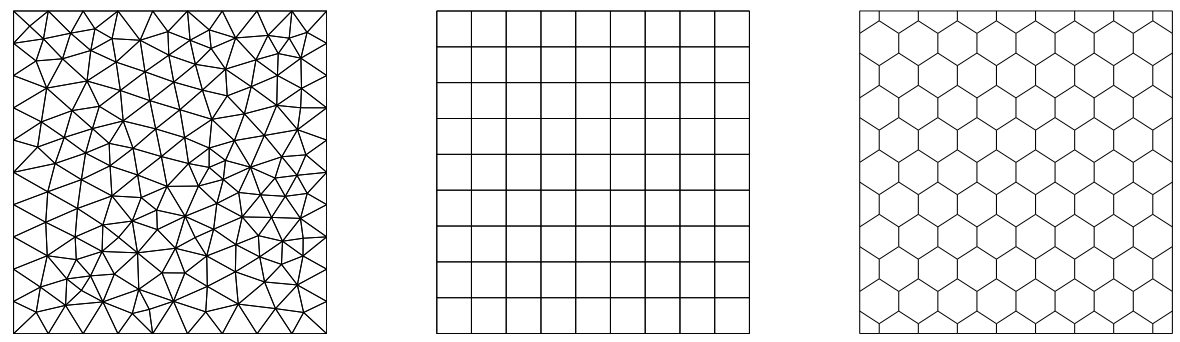

Fig. 1 Sample meshes: $\mathcal{T}_{h}^{1}$ (left), $\mathcal{T}_{h}^{2}$ (middle) and $\mathcal{T}_{h}^{3}$ (right). In all of them $N=9$.

Let us remark that for triangular and rectangular meshes like $\mathcal{T}_{h}^{1}$ and $\mathcal{T}_{h}^{2}$, respectively, the discrete spaces $\mathcal{V}_{h}$ coincide with those of the standard lowest-order RaviartThomas discretization. However, the resulting discrete problems are not the same. In fact, the matrices corresponding to the left-hand side of Problem 3 also coincide, but this does not happen with the matrices corresponding to right-hand side.

We report in Table 1 the scaled lowest eigenvalues $\widehat{\lambda}_{h i}:=\lambda_{h i} / \pi^{2}$ computed with the method analyzed in this paper. The table also includes estimated orders of convergence. The exact eigenvalues are also reported in the last column to allow for comparison.

It can be seen from Table 1 that the computed eigenvalues converge to the exact ones with an optimal quadratic order as predicted by the theory in almost all cases. The exception seems to be the computation of some of the eigenvalues with the hexagonal 
Table 1 Test 1 . Computed lowest eigenvalues $\widehat{\lambda}_{h i}, 1 \leq i \leq 5$, on different meshes with $\sigma_{E}=1$.

\begin{tabular}{|c|c|c|c|c|c|c|c|}
\hline $\mathcal{T}_{h}$ & $\widehat{\lambda}_{h i}$ & $N=19$ & $N=35$ & $N=53$ & $N=71$ & Order & $\lambda_{i}$ \\
\hline & $\widehat{\lambda}_{h 1}$ & 0.8248 & 0.8259 & 0.8262 & 0.8263 & 2.01 & 0.82645 \\
$\mathcal{T}_{h}^{1}$ & $\widehat{\lambda}_{h 2}$ & 0.9976 & 0.9993 & 0.9997 & 0.9998 & 2.00 & 1.00000 \\
& $\widehat{\lambda}_{h 3}$ & 1.8182 & 1.8240 & 1.8254 & 1.8259 & 2.01 & 1.82645 \\
& $\widehat{\lambda}_{h 4}$ & 3.2788 & 3.2978 & 3.3023 & 3.3039 & 2.02 & 3.30579 \\
& $\widehat{\lambda}_{h 5}$ & 3.9595 & 3.9883 & 3.9949 & 3.9972 & 2.03 & 4.00000 \\
\hline \multirow{3}{*}{$\mathcal{T}_{h}^{2}$} & $\widehat{\lambda}_{h 1}$ & 0.8200 & 0.8245 & 0.8256 & 0.8260 & 1.99 & 0.82645 \\
& $\widehat{\lambda}_{h 2}$ & 0.9896 & 0.9969 & 0.9987 & 0.9992 & 1.99 & 1.00000 \\
& $\widehat{\lambda}_{h 3}$ & 1.8096 & 1.8214 & 1.8243 & 1.8252 & 1.99 & 1.82645 \\
& $\widehat{\lambda}_{h 4}$ & 3.2047 & 3.2754 & 3.2925 & 3.2983 & 1.98 & 3.30579 \\
& $\widehat{\lambda}_{h 5}$ & 3.8389 & 3.9512 & 3.9786 & 3.9880 & 1.97 & 4.00000 \\
\hline \multirow{3}{*}{$\mathcal{T}_{h}^{3}$} & $\widehat{\lambda}_{h 1}$ & 0.8249 & 0.8260 & 0.8262 & 0.8263 & 1.98 & 0.82645 \\
& $\widehat{\lambda}_{h 2}$ & 0.9948 & 0.9982 & 0.9990 & 0.9993 & 1.56 & 1.00000 \\
& $\widehat{\lambda}_{h 3}$ & 1.8132 & 1.8220 & 1.8241 & 1.8249 & 1.63 & 1.82645 \\
& $\widehat{\lambda}_{h 4}$ & 3.2805 & 3.2979 & 3.3024 & 3.3039 & 1.98 & 3.30579 \\
& $\widehat{\lambda}_{h 5}$ & 3.9387 & 3.9823 & 3.9912 & 3.9946 & 1.84 & 4.00000 \\
\hline
\end{tabular}

meshes. In this case, although the computed eigenvalues are as good approximations to the exact ones as those computed with the other families of meshes, the order of convergence deteriorates mildly. We have observed from our numerical experiments that this can be avoided by choosing a smaller stability constant $\sigma_{E}$.

This can be clearly seen by comparing the lowest part of Table 1 with Table 2, where we report the result obtained with a smaller value of $\sigma_{E}$ and meshes $\mathcal{T}_{h}^{3}$. A more detailed discussion about the effect of the stability constant $\sigma_{E}$ appears in the following test.

Table 2 Test 1 . Computed lowest eigenvalues $\widehat{\lambda}_{h i}, 1 \leq i \leq 5$, on meshes $\mathcal{T}_{h}^{3}$ with $\sigma_{E}=2^{-4}$.

\begin{tabular}{|c|c|c|c|c|c|c|c|}
\hline $\mathcal{T}_{h}$ & $\widehat{\lambda}_{h i}$ & $N=19$ & $N=35$ & $N=53$ & $N=71$ & Order & $\lambda_{i}$ \\
\hline & $\widehat{\lambda}_{h 1}$ & 0.8294 & 0.8272 & 0.8268 & 0.8266 & 2.09 & 0.82645 \\
$\mathcal{T}_{h}^{3}$ & $\widehat{\lambda}_{h 2}$ & 1.0032 & 1.0009 & 1.0004 & 1.0002 & 2.15 & 1.00000 \\
& $\widehat{\lambda}_{h 3}$ & 1.8389 & 1.8297 & 1.8278 & 1.8272 & 2.12 & 1.82645 \\
& $\widehat{\lambda}_{h 4}$ & 3.3539 & 3.3179 & 3.3112 & 3.3088 & 2.09 & 3.30579 \\
& $\widehat{\lambda}_{h 5}$ & 4.0536 & 4.0149 & 4.0063 & 4.0034 & 2.09 & 4.00000 \\
\hline
\end{tabular}

Figure 2 shows plots of the computed eigenfunctions $\boldsymbol{w}_{h 1}$ and $\boldsymbol{w}_{h 3}$ corresponding to the first and third lowest eigenvalues, respectively. The figure also includes the corresponding pressure fluctuation $p_{h i}=-\operatorname{div} \boldsymbol{w}_{h i}, i=1,3$. In both cases, the eigenfunctions have been computed on an hexagonal mesh $\mathcal{T}_{h}^{3}$ with $N=27$ and stability constant $\sigma_{E}=1$.

\subsection{Test 2: Effect of the stability constant $\sigma_{E}$}

As was shown in the previous test, in some cases the quality of the computation can be affected by the choice of the stability constant $\sigma_{E}$. A similar behavior was observed in 

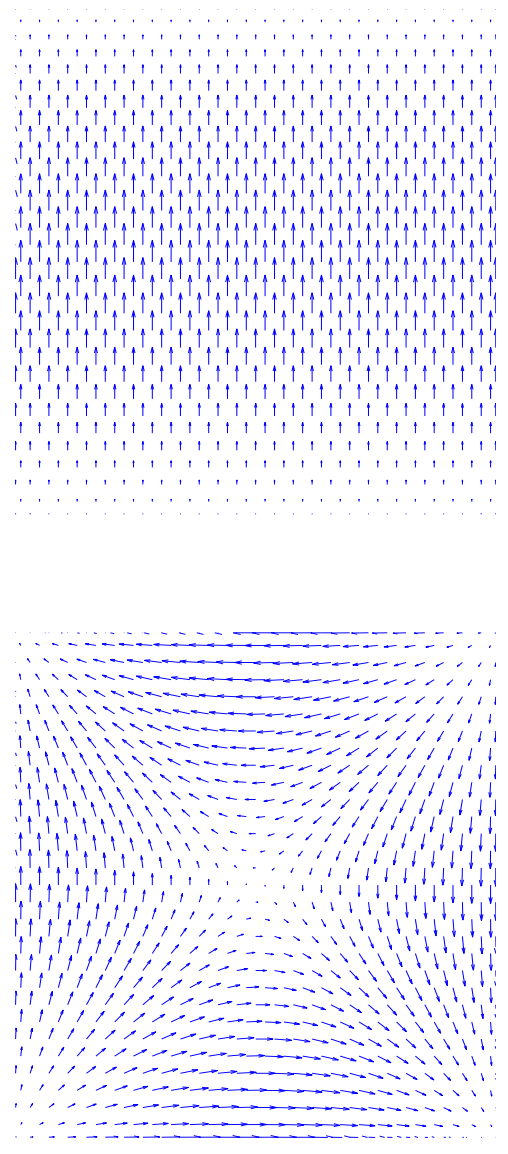
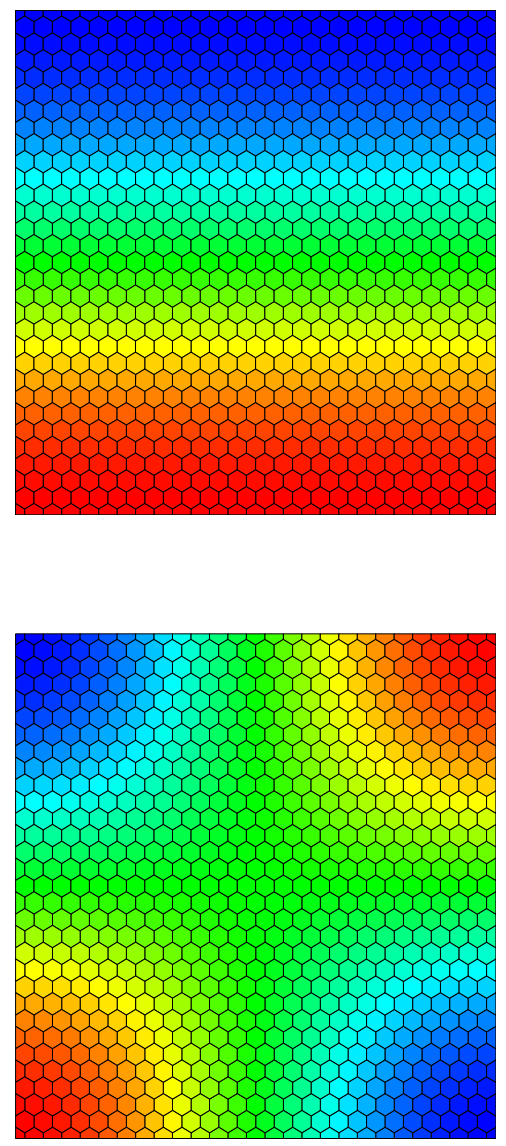

Fig. 2 Eigenfunctions of the acoustic problem corresponding to the first and third lowest eigenvalues: displacement field $\boldsymbol{w}_{h 1}$ (upper left), pressure fluctuation $p_{h 1}$, (upper right), displacement field $\boldsymbol{w}_{h 3}$ (bottom left), pressure fluctuation $p_{h 3}$ (bottom right).

other VEM for different eigenvalue problems. In particular, it was demonstrated in [32] that certain VEM discretizations of the Steklov eigenvalue problem introduces spurious eigenvalues which can be well separated from the physical spectrum by choosing appropriately the stability constant $\sigma_{E}$.

In the present case, no spurious eigenvalue was detected for any choice of the stability constant. However, for large values of $\sigma_{E}$, the eigenvalues computed with coarse meshes could be very poor. The aim of this test is to analyze the influence of the stability constant $\sigma_{E}$ on the computed spectrum.

We report in Table 3 the lowest eigenvalue computed with varying values of $\sigma_{E}$ on the family of meshes $\mathcal{T}_{h}^{2}$ (see Figure 2, middle). The table also includes the estimated order of convergence. 
Table 3 Test 2. The lowest eigenvalue $\widehat{\lambda}_{h 1}$ for $\sigma_{E}=0$ and $\sigma_{E}=2^{-k}$ with $-6 \leq k \leq 6$.

\begin{tabular}{|c|c|c|c|c|c|c|c|}
\hline$N$ & $\sigma_{E}=0$ & $\sigma_{E}=2^{-6}$ & $\sigma_{E}=2^{-5}$ & $\sigma_{E}=2^{-4}$ & $\sigma_{E}=2^{-3}$ & $\sigma_{E}=2^{-2}$ & $\sigma_{E}=2^{-1}$ \\
\hline 8 & 0.8482 & 0.8472 & 0.8463 & 0.8444 & 0.8406 & 0.8332 & 0.8187 \\
16 & 0.8318 & 0.8316 & 0.8313 & 0.8309 & 0.8300 & 0.8281 & 0.8245 \\
32 & 0.8278 & 0.8277 & 0.8277 & 0.8275 & 0.8273 & 0.8269 & 0.8260 \\
64 & 0.8268 & 0.8268 & 0.8268 & 0.8267 & 0.8267 & 0.8265 & 0.8263 \\
128 & 0.8265 & 0.8265 & 0.8265 & 0.8265 & 0.8265 & 0.8265 & 0.8264 \\
256 & 0.8265 & 0.8265 & 0.8265 & 0.8265 & 0.8265 & 0.8265 & 0.8264 \\
\hline Order & 2.00 & 2.00 & 2.00 & 2.00 & 2.00 & 2.00 & 2.00 \\
\hline$\lambda_{1}$ & 0.82645 & 0.82645 & 0.82645 & 0.82645 & 0.82645 & 0.82645 & 0.82645 \\
\hline$N$ & $\sigma_{E}=2^{0}$ & $\sigma_{E}=2^{1}$ & $\sigma_{E}=2^{2}$ & $\sigma_{E}=2^{3}$ & $\sigma_{E}=2^{4}$ & $\sigma_{E}=2^{5}$ & $\sigma_{E}=2^{6}$ \\
\hline 8 & 0.7912 & 0.7415 & 0.6586 & 0.5383 & 0.3943 & 0.2569 & 0.1513 \\
16 & 0.8174 & 0.8034 & 0.7770 & 0.7289 & 0.6487 & 0.5317 & 0.3907 \\
32 & 0.8242 & 0.8206 & 0.8135 & 0.7997 & 0.7735 & 0.7258 & 0.6463 \\
64 & 0.8259 & 0.8250 & 0.8233 & 0.8196 & 0.8125 & 0.7988 & 0.7726 \\
128 & 0.8263 & 0.8261 & 0.8256 & 0.8247 & 0.8229 & 0.8193 & 0.8123 \\
256 & 0.8264 & 0.8264 & 0.8262 & 0.8260 & 0.8256 & 0.8247 & 0.8229 \\
\hline Order & 1.99 & 1.97 & 1.94 & 1.90 & 1.82 & 1.70 & 1.55 \\
\hline$\lambda_{1}$ & 0.82645 & 0.82645 & 0.82645 & 0.82645 & 0.82645 & 0.82645 & 0.82645 \\
\hline
\end{tabular}

It can be seen from Table 3 that for values of the parameter $\sigma_{E} \leq 1$ the computed eigenvalues depend very mildly on this parameter. Moreover, this dependence becomes weaker, as the mesh is refined or $\sigma_{E}$ is taken smaller. In fact, it can be seen from this table that even the value $\sigma_{E}=0$ yields very accurate results, in spite of the fact that for such a value of the parameter the stability estimate and hence most of the proofs of the theoretical results do not hold. On the other hand, it can be seen from Table 3 that the numerical results depend much more significantly on this parameter $\sigma_{E}$ when it is chosen larger. In such a case, the results for coarse meshes are poorer and more refined meshes are needed for the computed eigenvalues to lie close to the exact ones.

This analysis suggests that the user of H(div) VEM for this kind of spectral problems has to be aware of the risk of degeneration of the eigenvalues for certain values of the stability constant $\sigma_{E}$. The way of minimizing this risk in this case is to take small values of $\sigma_{E}$ (what "small" means in a real problem will of course depend on the value of the physical constants).

\section{A Appendix}

We derive in this appendix optimal approximation properties for the $\mathrm{H}($ div) virtual elements with vanishing rotor introduced in Section 3. The main goal of this appendix will be to prove the error estimates stated in Lemmas 5 and 6 for the $\mathcal{V}_{h}$-interpolant defined by (8)-(9). Let us remark that these results could be useful for other applications as well.

Our first result, whose proof is quite straightforward, is a commuting diagram property and some consequences that follow from it. We recall that $P_{k}$ denotes the $\mathrm{L}^{2}(\Omega)$-orthogonal projection onto the subspace $\left\{q \in \mathrm{L}^{2}(\Omega):\left.q\right|_{E} \in \mathbb{P}_{k}(E) \quad \forall E \in \mathcal{T}_{h}\right\}$.

Lemma 5 Let $\boldsymbol{v} \in \mathcal{V}$ be such that $\boldsymbol{v} \in\left[\mathrm{H}^{t}(\Omega)\right]^{2}$ with $t>1 / 2$. Let $\boldsymbol{v}_{I} \in \mathcal{V}_{h}$ be its interpolant defined by (8)-(9). Then,

$$
\operatorname{div} \boldsymbol{v}_{I}=P_{k}(\operatorname{div} \boldsymbol{v}) \quad \text { in } \Omega .
$$

Consequently, for all $E \in \mathcal{T}_{h},\left\|\operatorname{div} \boldsymbol{v}_{I}\right\|_{0, E} \leq\|\operatorname{div} \boldsymbol{v}\|_{0, E}$ and, if $\left.\operatorname{div} \boldsymbol{v}\right|_{E} \in \mathrm{H}^{r}(E)$ with $r \geq 0$, then

$$
\left\|\operatorname{div} \boldsymbol{v}-\operatorname{div} \boldsymbol{v}_{I}\right\|_{0, E} \leq C h_{E}^{\min \{r, k+1\}}|\operatorname{div} \boldsymbol{v}|_{r, E}
$$


Proof As a consequence of (8)-(9), for every element $E$ and for every $q \in \mathbb{P}_{k}(E)$

$$
\int_{E} \operatorname{div}\left(\boldsymbol{v}-\boldsymbol{v}_{I}\right) q=\int_{E}\left(\boldsymbol{v}-\boldsymbol{v}_{I}\right) \cdot \nabla q+\int_{\partial E}\left(\boldsymbol{v}-\boldsymbol{v}_{I}\right) \cdot \boldsymbol{n} q d s=0 .
$$

Since $\operatorname{div} \boldsymbol{v}_{I} \in \mathbb{P}_{k}(E)$, we have that

$$
\operatorname{div} \boldsymbol{v}_{I}=P_{k}(\operatorname{div} \boldsymbol{v}) \text { in } E .
$$

Therefore,

$$
\left\|\operatorname{div} \boldsymbol{v}_{I}\right\|_{0, E} \leq\|\operatorname{div} \boldsymbol{v}\|_{0, E} .
$$

Additionally, if $\left.\operatorname{div} \boldsymbol{v}\right|_{E} \in \mathrm{H}^{r}(E)$ with $r$ a non-negative integer, as a consequence of [19, Lemma 4.3.8], we have that for every $E \in \mathcal{T}_{h}$

$$
\left\|\operatorname{div} \boldsymbol{v}-\operatorname{div} \boldsymbol{v}_{I}\right\|_{0, E} \leq C h_{E}^{\min \{r, k+1\}}|\operatorname{div} \boldsymbol{v}|_{r, E} .
$$

Thus, the second estimate of the lemma follows by standard Banach space interpolation.

In order to prove Lemma 6 about the $\mathrm{L}^{2}(\Omega)$ approximation property of this interpolant, we need several previous results. We begin with the following local trace estimate on polygons.

Lemma 14 Let $\boldsymbol{v} \in \mathcal{V}$ and $E \in \mathcal{T}_{h}$ such that $\left.\boldsymbol{v}\right|_{E} \in\left[\mathrm{H}^{t}(E)\right]^{2}$ with $t \in(1 / 2,1]$. Then, there exists $C>0$ such that

$$
\|\boldsymbol{v}\|_{0, \partial E} \leq C\left(h_{E}^{-1 / 2}\|\boldsymbol{v}\|_{0, E}+h_{E}^{t-1 / 2}|\boldsymbol{v}|_{t, E}\right) .
$$

Proof Consider the triangulation $\mathcal{T}_{h}^{E}$ of the element $E$ obtained by joining each vertex of $E$ with the midpoint of the ball with respect to which $E$ is star-shaped. Since we are assuming that the meshes satisfy $\mathbf{A}_{1}$ and $\mathbf{A}_{\mathbf{2}}$, the triangles $T \in \mathcal{T}_{h}^{E}$ have a shape ratio (i.e., the quotient between outer and inner diameters) bounded above by a constant that only depends on $C_{\mathcal{T}}$. Moreover, each triangle $T \in \mathcal{T}_{h}^{E}$ has one edge on $\partial E$. Hence, a scaling argument and a trace inequality in the reference triangular element allow us to conclude the proof.

In order to prove an $\mathrm{L}^{2}(\Omega)$ error estimate for the interpolant $\boldsymbol{v}_{I}$, we will introduce a basis of $\mathcal{V}_{h}^{E}$ dual to the degrees of freedom (1)-(2).

Let $E \in \mathcal{T}_{h}$ with edges $e_{1}, \ldots, e_{N_{E}}$ and $F: E \longrightarrow \widehat{E}$ be an affine mapping of the form

$$
F\left(\begin{array}{l}
x \\
y
\end{array}\right):=\frac{1}{h_{E}}\left(\begin{array}{l}
x-x_{E} \\
y-y_{E}
\end{array}\right)=:\left(\begin{array}{l}
\widehat{x} \\
\widehat{y}
\end{array}\right)
$$

where $\boldsymbol{x}_{E}=\left(x_{E}, y_{E}\right)^{T}$ is the center of the ball with respect to which $E$ is star-shaped according to assumption $\mathbf{A}_{2}$. Note that $\widehat{E}:=F(E)$ has diameter 1. Moreover, $F$ maps the above mentioned ball onto a ball of radius $C_{\mathcal{T}}$ with $0<C_{\mathcal{T}} \leq 1$ and $C_{\mathcal{T}}$ independent of $h_{E}$, Moreover, $\widehat{E}$ is star-shaped with respect to each point of this ball.

We define the following basis of $\mathbb{P}_{k}(E)$ :

$$
\begin{aligned}
& p_{0}(x, y):=1, \\
& p_{s}(x, y):=\frac{\left(x-x_{E}\right)^{\alpha_{1}}\left(y-y_{E}\right)^{\alpha_{2}}}{h_{E}^{\alpha_{1}+\alpha_{2}}}+C_{s}, \quad \alpha_{1}, \alpha_{2} \in \mathbb{N}, \quad 0<\alpha_{1}+\alpha_{2} \leq k,
\end{aligned}
$$

with the constant $C_{s} \in \mathbb{R}$ such that $\int_{E} p_{s}=0$. We have associated above each $s=1, \ldots, \widetilde{N}:=$ $\operatorname{dim}\left(\mathbb{P}_{k}(E)\right)-1$ with one particular couple $\left(\alpha_{1}, \alpha_{2}\right)$, by fixing a particular ordering of these couples. Therefore, the set $\left\{p_{0}, p_{1}, \ldots, p_{\widetilde{N}}\right\}$ is a basis for $\mathbb{P}_{k}(E)$ that satisfies $\int_{E} p_{s}=0$ for $s=1, \ldots, \widetilde{N}$. Let now $\widehat{p}_{s}:=p_{s} \circ F^{-1}$ be defined in $\widehat{E}$. Then, for the particular $\left(\alpha_{1}, \alpha_{2}\right)$ associated with $s$, we have that $\widehat{p}_{s}(\widehat{x}, \widehat{y})=\widehat{x}^{\alpha_{1}} \widehat{y}^{\alpha_{2}}+C_{s}$. Moreover, since $|E|=h_{E}^{2}|\widehat{E}|$, we have

$$
C_{s}=-\frac{1}{|E|} \int_{E} \frac{\left(x-x_{E}\right)^{\alpha_{1}}\left(y-y_{E}\right)^{\alpha_{2}}}{h_{E}^{\alpha_{1}+\alpha_{2}}} d x d y=-\frac{1}{|\widehat{E}|} \int_{\widehat{E}} \widehat{x}^{\alpha_{1}} \widehat{y}^{\alpha_{2}} d \widehat{x} d \widehat{y} .
$$


As a consequence, note that $\left|C_{s}\right| \leq 1$ and, hence, $\left\|p_{s}\right\|_{\infty, E}=\left\|\widehat{p}_{s}\right\|_{\infty, \widehat{E}} \leq 2, s=0, \ldots, \widetilde{N}$.

For each edge $e_{l}$ of $E\left(l=1, \ldots, N_{E}\right)$, let $T_{l}$ be the affine function mapping $\widehat{e}:=[-1,1]$ onto $e_{l}$. We define $q_{l}^{i}:=\widehat{q}^{i} \circ T_{l}^{-1}(i=1, \ldots, k)$ with $\widehat{q}^{i}$ being the Legendre polynomials on $[-1,1]$ normalized by $\widehat{q}^{i}(1)=1$. Then, $\left\{q_{l}^{0}, \ldots, q_{l}^{k}\right\}$ is a basis of $\mathbb{P}_{k}\left(e_{l}\right)$ which satisfies $q_{l}^{0}=1, \int_{e_{l}} q_{l}^{i} q_{l}^{j} d s=\delta_{i j}$, $i, j=1, \ldots, k$, and $\left\|q_{l}^{i}\right\|_{\infty, e_{l}}=1$. Note that, in particular, $\int_{e_{l}} q_{l}^{i} d s=0, i=1, \ldots, k$.

Therefore,

$$
\left\{q_{l}^{i}\right\}_{i=0, \ldots, k, l=1, \ldots, N_{E}} \quad \text { and } \quad\left\{p_{s}\right\}_{s=1, \ldots, \widetilde{N}}
$$

are bases for the spaces of test functions appearing in the degrees of freedom (8) and (9), respectively. Next, we introduce a set of dual basis functions for $\mathcal{V}_{h}^{E}$ :

$$
\left\{\boldsymbol{\varphi}_{l}^{i}\right\}_{i=0, \ldots, k, l=1, \ldots, N_{E}} \cup\left\{\widetilde{\boldsymbol{\varphi}}^{s}\right\}_{s=1, \ldots, \widetilde{N}}
$$

The first ones, $\boldsymbol{\varphi}_{l}^{i}$, are the "boundary basis functions" determined by

$$
\begin{aligned}
& \varphi_{l}^{i} \in \mathcal{V}_{h}^{E}, \\
& \int_{e_{m}}\left(\varphi_{l}^{i} \cdot \boldsymbol{n}\right) q_{m}^{j} d s=\delta_{l m} \delta_{i j}, \quad m=1, \ldots, N_{E}, \quad j=0, \ldots, k, \\
& \int_{E}\left(\operatorname{div} \varphi_{l}^{i}\right) p_{r}=0, \quad r=1, \ldots, \tilde{N} .
\end{aligned}
$$

Note that these boundary basis functions use two indexes, $i$ and $l$, one for the moment and the other for the edge. On the other hand, note also that as a consequence of $(23)-(24) \boldsymbol{\varphi}_{l}^{i} \cdot \boldsymbol{n}=0$ on $\partial E \backslash e_{l}$ The second kind of functions in $(22), \widetilde{\varphi}^{s}$, are the "internal basis functions" determined by

$$
\begin{aligned}
& \widetilde{\varphi}^{s} \in \mathcal{V}_{h}^{E}, \\
& \left.\widetilde{\boldsymbol{\varphi}}^{s}\right|_{\partial E} \cdot \boldsymbol{n}=0, \\
& \int_{E}\left(\operatorname{div} \widetilde{\boldsymbol{\varphi}}^{s}\right) p_{r}=\delta_{s r}, \quad r=1, \ldots, \widetilde{N} .
\end{aligned}
$$

Remark 5 Since $\operatorname{div} \varphi_{l}^{i} \in \mathbb{P}_{k}(E)=\operatorname{span}\left\{1, p_{1}, \ldots, p_{s}\right\}$ and $\int_{E} p_{s}=0$ for $s=1, \ldots, \tilde{N}$, equation (25) implies that $\operatorname{div} \varphi_{l}^{i}$ has to be constant. Therefore,

$$
\operatorname{div} \boldsymbol{\varphi}_{l}^{i}=\frac{1}{|E|} \int_{E} \operatorname{div} \boldsymbol{\varphi}_{l}^{i}=\frac{1}{|E|} \int_{\partial E} \boldsymbol{\varphi}_{l}^{i} \cdot \boldsymbol{n} d s
$$

Moreover, thanks to (24), we have that

$$
\int_{\partial E} \boldsymbol{\varphi}_{l}^{i} \cdot \boldsymbol{n} d s=\sum_{m=1}^{N_{E}} \int_{e_{m}}\left(\boldsymbol{\varphi}_{l}^{i} \cdot \boldsymbol{n}\right) q_{m}^{0} d s=\sum_{m=1}^{N_{E}} \delta_{l m} \delta_{i 0}=\delta_{i 0} .
$$

Then,

$$
\operatorname{div} \varphi_{l}^{i}=\frac{\delta_{i 0}}{|E|}
$$

Next goal is to prove that all the functions in (22) are bounded uniformly in $h$. We begin with the boundary basis functions.

Lemma 15 There exists $C>0$ such that $\left\|\varphi_{l}^{i}\right\|_{0, E} \leq C$ for $l=1, \ldots, N_{E}$ and $i=0, \ldots, k$.

Proof Since $\varphi_{l}^{i} \in \mathcal{V}_{h}^{E}$, we know that $\operatorname{rot} \varphi_{l}^{i}=0$. Therefore, there exists $\gamma \in \mathrm{H}^{1}(E)$ such that $\varphi_{l}^{i}=\nabla \gamma$. Hence, from the remark above and (24), we have that $\gamma$ is a solution of the following problem:

$$
\left\{\begin{array}{l}
\Delta \gamma=\frac{\delta_{i 0}}{|E|} \quad \text { in } E \\
\frac{\partial \gamma}{\partial \boldsymbol{n}}=\boldsymbol{\varphi}_{l}^{i} \cdot \boldsymbol{n} \\
\int_{E} \gamma=0 .
\end{array}\right.
$$


It is easy to check that these Neumann problems are compatible. Therefore,

$$
\int_{E} \nabla \gamma \cdot \nabla \zeta=\int_{\partial E}\left(\boldsymbol{\varphi}_{l}^{i} \cdot \boldsymbol{n}\right) \zeta d s-\int_{E} \frac{\delta_{i 0}}{|E|} \zeta=\int_{e_{l}}\left(\boldsymbol{\varphi}_{l}^{i} \cdot \boldsymbol{n}\right) \zeta d s \quad \forall \zeta \in \mathrm{H}^{1}(E): \int_{E} \zeta=0 .
$$

Now, taking $\zeta=\gamma$, we obtain

$$
\begin{aligned}
\left\|\boldsymbol{\varphi}_{l}^{i}\right\|_{0, E}^{2} & =\|\nabla \gamma\|_{0, E}^{2} \leq\left\|\boldsymbol{\varphi}_{l}^{i} \cdot \boldsymbol{n}\right\|_{0, e_{l}}\|\gamma\|_{0, e_{l}} \\
& \leq C\left\|\boldsymbol{\varphi}_{l}^{i} \cdot \boldsymbol{n}\right\|_{0, e_{l}}\left(h_{E}^{-1 / 2}\|\gamma\|_{0, E}+h_{E}^{1 / 2}\|\nabla \gamma\|_{0, E}\right) \\
& \leq C h_{E}^{1 / 2}\left\|\boldsymbol{\varphi}_{l}^{i} \cdot \boldsymbol{n}\right\|_{0, e_{l}}\|\nabla \gamma\|_{0, E},
\end{aligned}
$$

where we have used Lemma 14 with $t=1$, the generalized Poincaré inequality and a scaling argument. Now, because of (24) with $m=l$ and the orthogonality property of Legendre polynomials, $\left.\boldsymbol{\varphi}_{l}^{i} \cdot \boldsymbol{n}\right|_{e_{l}}=\left(\int_{e_{l}}\left(q_{l}^{i}\right)^{2} d s\right)^{-1} q_{l}^{i}$. Therefore,

$$
\left\|\boldsymbol{\varphi}_{l}^{i} \cdot \boldsymbol{n}\right\|_{0, e_{l}}^{2}=\left(\int_{e_{l}}\left(q_{l}^{i}\right)^{2} d s\right)^{-1}=\frac{1}{h_{E}}\left(\int_{\widehat{e}}\left(\widehat{q}^{i}\right)^{2} d \widehat{s}\right)^{-1} .
$$

Thus, from the last two estimates we derive that $\left\|\varphi_{l}^{i}\right\|_{0, E} \leq C$ and we end the proof.

Next, we show a similar result for the internal basis functions.

Lemma 16 There exists $C>0$ such that $\left\|\widetilde{\varphi}^{s}\right\|_{0, E} \leq C$ for $s=1, \ldots, \tilde{N}$.

Proof Since $\widetilde{\varphi}^{s} \in \mathcal{V}_{h}^{E}$, there exists $\gamma \in \mathrm{H}^{1}(E)$ such that $\widetilde{\varphi}^{s}=\nabla \gamma$. Hence, by virtue of (27), we have that $\gamma$ is a solution of the following well posed Neumann problem:

$$
\begin{cases}\Delta \gamma=-\operatorname{div} \widetilde{\boldsymbol{\varphi}}^{s} & \text { in } E, \\ \frac{\partial \gamma}{\partial \boldsymbol{n}}=0 & \text { on } \partial E \\ \int_{E} \gamma=0 . & \end{cases}
$$

Therefore,

$$
\int_{E} \nabla \gamma \cdot \nabla \zeta=-\int_{E} \psi^{s} \zeta \quad \forall \zeta \in \mathrm{H}^{1}(E): \int_{E} \zeta=0,
$$

where $\psi^{s}:=\operatorname{div} \widetilde{\varphi}^{s}$. Now, taking $\zeta=\gamma$ and using the generalized Poincaré inequality and a scaling argument, we have that

$$
\left\|\widetilde{\varphi}^{s}\right\|_{0, E}^{2}=\|\nabla \gamma\|_{0, E}^{2} \leq C\left\|\psi^{s}\right\|_{0, E}\|\gamma\|_{0, E} \leq C h_{E}\left\|\psi^{s}\right\|_{0, E}\|\nabla \gamma\|_{0, E}
$$

Thus,

$$
\left\|\widetilde{\varphi}^{s}\right\|_{0, E} \leq C h_{E}\left\|\psi^{s}\right\|_{0, E} .
$$

On the other hand, since $\psi^{s} \in \mathbb{P}_{k}(E)$, it is easy to check that

$$
h_{E}\left\|\psi^{s}\right\|_{0, E} \leq C h_{E}^{2}\left\|\psi^{s}\right\|_{\infty, E}=C h_{E}^{2}\left\|\widehat{\psi}^{s}\right\|_{\infty, \widehat{E}},
$$

where $\widehat{\psi}^{s}:=\left(\psi^{s} \circ F^{-1}\right) \in \mathbb{P}_{k}(\widehat{E})$.

For $\widehat{\psi}^{s} \in \mathbb{P}_{k}(\widehat{E})$, we write $\widehat{\psi}^{s}=\sum_{i=1}^{\widetilde{N}} \beta_{i}^{s} \widehat{p}_{i}$ and, since $\left\|\widehat{p}_{i}\right\|_{\infty, \widehat{E}} \leq 2$, we have that

$$
\left\|\widehat{\psi}^{s}\right\|_{\infty, \widehat{E}} \leq \max _{1 \leq i \leq \widetilde{N}}\left|\beta_{i}^{s}\right| \sum_{i=1}^{\widetilde{N}}\left\|\widehat{p}_{i}\right\|_{\infty, \widehat{E}} \leq C \max _{1 \leq i \leq \widetilde{N}}\left|\beta_{i}^{s}\right|
$$


Now, from (28), a change of variables from $E$ to $\widehat{E}$ yields

$$
\int_{\widehat{E}} \widehat{\psi}^{s} \widehat{p}_{r}=h_{E}^{-2} \delta_{s r}, \quad r=1, \ldots, \widetilde{N}
$$

which can be written as

$$
\sum_{i=1}^{\widetilde{N}} \beta_{i}^{s} \int_{\widehat{E}} \widehat{p}_{i} \widehat{p}_{r}=h_{E}^{-2} \delta_{s r}, \quad r=1, \ldots, \widetilde{N}
$$

Let

$$
\mathbf{M}=\left(m_{i r}\right) \in \mathbb{R}^{\widetilde{N} \times \widetilde{N}} \quad \text { with } m_{i r}:=\int_{\widehat{E}} \widehat{p}_{i} \widehat{p}_{r}, \quad i, r=1, \ldots, \widetilde{N}
$$

Therefore, from (32), if $\mathbf{M}$ is invertible, then $\boldsymbol{\beta}^{s}=\left(\beta_{1}^{s} \cdots \beta_{\widetilde{N}}^{s}\right)^{T}$ is equal to $h_{E}^{-2}$ times the $s$-th column of $\mathbf{M}^{-1}$.

Next, we will show that $\mathbf{M}$ is invertible and that its inverse is bounded uniformly in $h$. With this aim, note that the polygon $\widehat{E}$ is uniquely defined by the vector $\left(\left(\widehat{x}_{1}, \widehat{y}_{1}\right), \ldots,\left(\widehat{x}_{N_{E}}, \widehat{y}_{N_{E}}\right)\right) \in \mathbb{R}^{2 N_{E}}$ that collects the coordinates of its (ordered) vertexes. Let $U \subset \mathbb{R}^{2 N_{E}}$, be the set of all possible values of these coordinates such that the mesh regularity assumptions $\mathbf{A}_{\mathbf{1}}$ and $\mathbf{A}_{\mathbf{2}}$ are satisfied. Since the diameter of $\widehat{E}$ is equal to $1, U$ is a bounded set. On the other hand, the constraints that arise from hypotheses $\mathbf{A}_{1}$ and $\mathbf{A}_{2}$ yield that $U$ is a closed set. Therefore $U$ is compact.

The function from $U$ into $\mathbb{R}^{\widetilde{N} \times \widetilde{N}}$ that maps the coordinates of the vertexes of $\widehat{E}$ into the entries of the matrix $\mathbf{M}$ is a continuous function. Moreover, for any coordinates in $U, \widehat{E}$ satisfies $\mathbf{A}_{\mathbf{1}}$ and $\mathbf{A}_{2}$ and, hence, it contains a ball of radius $C_{\mathcal{T}}$. Let us show that this implies that $\mathbf{M}$ has to be positive definite. In fact, given $\alpha \in \mathbb{R}^{\widetilde{N}}, \alpha^{T} \mathbf{M} \alpha=\int_{\widehat{E}}\left|\sum_{r=1}^{\widetilde{N}} \alpha_{r} \widehat{p}_{r}\right|^{2} \geq 0$ and the equality holds only if $\sum_{r=1}^{\widetilde{N}} \alpha_{r} \widehat{p}_{r}$ vanishes a.e. in $\widehat{E}$, which in turn implies that $\alpha$ has to vanish (since $\widehat{E}$ contains a ball of radius $C_{\mathcal{T}}>0$ ). Thus, $\mathbf{M}$ is positive definite and hence invertible. Therefore, taking also into account the continuity of the mapping $\mathbf{M} \longmapsto \mathbf{M}^{-1}$ for invertible matrices, we conclude that the mapping

$$
U \ni\left(\left(\widehat{x}_{1}, \widehat{y}_{1}\right), \ldots,\left(\widehat{x}_{N_{E}}, \widehat{y}_{N_{E}}\right)\right) \longmapsto \mathbf{M}^{-1} \in \mathbb{R}^{\tilde{N} \times \tilde{N}}
$$

is well defined and continuous and, hence, bounded above in the compact set $U$. Consequently, from (32),

which recalling (31) yields

$$
\left\|\boldsymbol{\beta}^{s}\right\|_{\infty} \leq C h_{E}^{-2}
$$

$$
\left\|\widehat{\psi}^{s}\right\|_{\infty, \widehat{E}} \leq C h_{E}^{-2} .
$$

Let us remark that, in principle, the constant $C$ above depends on the number $N_{E}$ of vertexes of $E$. However, by virtue of assumption $\mathbf{A}_{\mathbf{1}}$, this number is bounded above in terms of $C_{\mathcal{T}}$. Therefore, $N_{E}$ can take only a finite number of possible values and, hence, (33) holds true with $C$ only depending on $C_{\mathcal{T}}$. Thus, we conclude the proof by combining (29), (30) and (33).

Now, we are in a position to prove $\mathrm{L}^{2}(\Omega)$ error estimates for the $\mathcal{V}_{h}$-interpolant.

Lemma 6 Let $\boldsymbol{v} \in \mathcal{V}$ be such that $\boldsymbol{v} \in\left[\mathrm{H}^{t}(\Omega)\right]^{2}$ with $t>1 / 2$. Let $\boldsymbol{v}_{I} \in \mathcal{V}_{h}$ be its interpolant defined by (8)-(9). Let $E \in \mathcal{T}_{h}$. If $1 \leq t \leq k+1$, then

$$
\left\|\boldsymbol{v}-\boldsymbol{v}_{I}\right\|_{0, E} \leq C h_{E}^{t}|\boldsymbol{v}|_{t, E}
$$

whereas, if $1 / 2<t \leq 1$, then

$$
\left\|\boldsymbol{v}-\boldsymbol{v}_{I}\right\|_{0, E} \leq C\left(h_{E}^{t}|\boldsymbol{v}|_{t, E}+h_{E}\|\operatorname{div} \boldsymbol{v}\|_{0, E}\right)
$$


Proof First, we consider the case $1 \leq t \leq k+1$. The first step is to bound $\left\|\boldsymbol{v}_{I}\right\|_{0, E}$. Since $\boldsymbol{v}_{I} \in \mathcal{V}_{h}^{E}$, thanks to (23)-(28) we write it in the basis (22) as follows:

$$
\boldsymbol{v}_{I}=\sum_{l=1}^{N_{E}} \sum_{i=0}^{k}\left(\int_{e_{l}}(\boldsymbol{v} \cdot \boldsymbol{n}) q_{l}^{i} d s\right) \boldsymbol{\varphi}_{l}^{i}+\sum_{s=1}^{\tilde{N}}\left(\int_{E}(\operatorname{div} \boldsymbol{v}) p_{s}\right) \widetilde{\boldsymbol{\varphi}}^{s} .
$$

Therefore, from Lemmas 15 and 16 we have

$$
\left\|\boldsymbol{v}_{I}\right\|_{0, E} \leq C\left(\sum_{l=1}^{N_{E}} \sum_{i=0}^{k}\left|\int_{e_{l}}(\boldsymbol{v} \cdot \boldsymbol{n}) q_{l}^{i} d s\right|+\sum_{s=1}^{\widetilde{N}}\left|\int_{E}(\operatorname{div} \boldsymbol{v}) p_{s}\right|\right) .
$$

Then, by using that $\left\|q_{l}^{i}\right\|_{\infty, e_{l}}=1$ for $i=1, \ldots, k$ and $l=1, \ldots, N_{E},\left\|p_{s}\right\|_{\infty, E} \leq C$ for $s=1, \ldots, \widetilde{N}$, the Cauchy-Schwarz inequality and Lemma 14, we obtain

$$
\begin{aligned}
\left\|\boldsymbol{v}_{I}\right\|_{0, E} & \leq C\left(h_{E}^{1 / 2}\|\boldsymbol{v}\|_{0, \partial E}\left\|q_{l}^{i}\right\|_{\infty, e_{l}}+\widetilde{N} h_{E}\|\operatorname{div} \boldsymbol{v}\|_{0, E}\left\|p_{s}\right\|_{\infty, E}\right) \\
& \leq C\left(\|\boldsymbol{v}\|_{0, E}+h_{E}|\boldsymbol{v}|_{1, E}+h_{E}\|\operatorname{div} \boldsymbol{v}\|_{0, E}\right) \\
& \leq C\left(\|\boldsymbol{v}\|_{0, E}+h_{E}|\boldsymbol{v}|_{1, E}\right) .
\end{aligned}
$$

Now, for all $\boldsymbol{v}_{k} \in\left[\mathbb{P}_{k}(E)\right]^{2}$ we note that $\left(\boldsymbol{v}_{k}\right)_{I}=\boldsymbol{v}_{k}$ and, hence, using the above estimate for $\boldsymbol{v}-\boldsymbol{v}_{k}$, we write

$$
\left\|\boldsymbol{v}-\boldsymbol{v}_{I}\right\|_{0, E}=\left\|\boldsymbol{v}-\boldsymbol{v}_{k}-\left(\boldsymbol{v}-\boldsymbol{v}_{k}\right)_{I}\right\|_{0, E} \leq\left\|\boldsymbol{v}-\boldsymbol{v}_{k}\right\|_{0, E}+C\left(\left\|\boldsymbol{v}-\boldsymbol{v}_{k}\right\|_{0, E}+h_{E}\left|\boldsymbol{v}-\boldsymbol{v}_{k}\right|_{1, E}\right) .
$$

Thus, by choosing $\boldsymbol{v}_{k}$ as in [6, Proposition 4.2], we have that $\left\|\boldsymbol{v}-\boldsymbol{v}_{k}\right\|_{0, E}+h_{E}\left|\boldsymbol{v}-\boldsymbol{v}_{k}\right|_{1, E} \leq$ $C h_{E}^{t}|\boldsymbol{v}|_{t, E}$, which together with the above inequality allow us to conclude (34).

Next, we consider the case $1 / 2<t \leq 1$. Using the same arguments as above, we obtain in this case instead of (36),

$$
\left\|\boldsymbol{v}_{I}\right\|_{0, E} \leq C\left(\|\boldsymbol{v}\|_{0, E}+h_{E}^{t}|\boldsymbol{v}|_{t, E}+h_{E}\|\operatorname{div} \boldsymbol{v}\|_{0, E}\right) .
$$

Therefore, repeating again the arguments above with $\boldsymbol{v}_{0} \in\left[\mathbb{P}_{0}(E)\right]^{2}$ instead of $\boldsymbol{v}_{k}$, we have

$$
\begin{aligned}
\left\|\boldsymbol{v}-\boldsymbol{v}_{I}\right\|_{0, E} & \leq\left\|\boldsymbol{v}-\boldsymbol{v}_{0}-\left(\boldsymbol{v}-\boldsymbol{v}_{0}\right)_{I}\right\|_{0, E} \\
& \leq\left\|\boldsymbol{v}-\boldsymbol{v}_{0}\right\|_{0, E}+C\left(\left\|\boldsymbol{v}-\boldsymbol{v}_{0}\right\|_{0, E}+h_{E}^{t}|\boldsymbol{v}|_{t, E}+h_{E}\|\operatorname{div} \boldsymbol{v}\|_{0, E}\right) \\
& \leq C\left(h_{E}^{t}|\boldsymbol{v}|_{t, E}+h_{E}\|\operatorname{div} \boldsymbol{v}\|_{0, E}\right),
\end{aligned}
$$

where we have used again [6, Proposition 4.2]. Thus, the proof is complete.

Remark 6 Estimate (35) can be improved for $k=0$ and $1 / 2<t \leq 1$. In fact, in such a case, the interpolant $\boldsymbol{v}_{I} \in \mathcal{V}_{h}$ is defined only by (8). Hence,

$$
\boldsymbol{v}_{I}=\sum_{l=1}^{N_{E}}\left(\int_{e_{l}}(\boldsymbol{v} \cdot \boldsymbol{n}) q_{l}^{0} d s\right) \boldsymbol{\varphi}_{l}^{0}
$$

and repeating the arguments above we obtain

$$
\left\|\boldsymbol{v}_{I}\right\|_{0, E} \leq C\left(\|\boldsymbol{v}\|_{0, E}+h_{E}^{t}|\boldsymbol{v}|_{t, E}\right)
$$

instead of (37), which leads to

$$
\left\|\boldsymbol{v}-\boldsymbol{v}_{I}\right\|_{0, E} \leq C h_{E}^{t}|\boldsymbol{v}|_{t, E}
$$




\section{References}

1. Ahmad, B., Alsaedi, A., Brezzi, F., Marini, L. D., Russo, A.: Equivalent projectors for virtual element methods. Comput. Math. Appl. 66, 376-391 (2013).

2. Antonietti, P. F., Beirão da Veiga, L., Mora, D., Verani, M.: A stream virtual element formulation of the Stokes problem on polygonal meshes. SIAM J. Numer. Anal. 52, 386-404 (2014).

3. Antonietti, P. F., Houston, P., Sarti, M., Verani, M.: Multigrid algorithms for $h p$-version interior penalty discontinuous Galerkin methods on polygonal and polyhedral meshes. Preprint arXiv:1412.0913 [math.NA] (2014).

4. Ayuso de Dios, B., Lipnikov, K., Manzini, G.: The nonconforming virtual element method: Preprint arXiv:1405.3741 [math.NA] (2014).

5. Babuška, I., Osborn, J.: Eigenvalue problems In: Ciarlet, P. G., Lions, J. L. (eds.) Handbook of Numerical Analysis, Vol. II, pp. 641-787. North-Holland, Amsterdam (1991).

6. Beirão da Veiga, L., Brezzi, F., Cangiani, A., Manzini, G., Marini, L. D., Russo, A.: Basic principles of virtual element methods. Math. Models Methods Appl. Sci. 23, 199-214 (2013).

7. Beirão da Veiga, L., Brezzi, F., Marini, L. D., Russo, A.: The hitchhiker's guide to the virtual element method. Math. Models Methods Appl. Sci. 24, 1541-1573 (2014).

8. Beirão da Veiga, L., Brezzi, F., Marini, L. D., Russo, A.: Mixed virtual element methods for general second order elliptic problems on polygonal meshes. Preprint arXiv:1506.07328 [math.NA] (2015).

9. Beirão da Veiga, L., Lipnikov, K., Manzini, G.: The Mimetic Finite Difference Method for Elliptic Problems. Springer, MS\&A, vol. 11 (2014).

10. Beirão da Veiga, L., Lovadina, C., Mora, D.: A virtual element method for elastic and inelastic problems on polytope meshes. Comput. Methods Appl. Mech. Engrg. 295, 327-346 (2015).

11. Benedetto, M. F., Berrone, S., Pieraccini, S., Scialò, S.: The virtual element method for discrete fracture network simulations. Comput. Methods Appl. Mech. Engrg. 280, 135-156 (2014).

12. Bermúdez, A., Durán, R., Muschietti, M.A., Rodríguez, R., Solomin, J.: Finite element vibration analysis of fluid-solid systems without spurious modes. SIAM J. Numer. Anal. 32, 1280-1295 (1995).

13. Bermúdez, A., Durán, R., Rodríguez, R., Solomin, J.: Finite element analysis of a quadratic eigenvalue problem arising in dissipative acoustics. SIAM J. Numer. Anal. 38, 267-291 (2000).

14. Bermúdez, A., Gamallo, P., Hervella-Nieto, L., Rodríguez, R., Santamarina, D.: Fluid-structure acoustic interaction. In: Marburg, S., Nolte, B. (eds.) Computational Acoustics of Noise Propagation in Fluids. Finite and Boundary Element Methods. Springer, Chap. 9, pp. 253-286 (2008).

15. Bermúdez, A., Hervella-Nieto, L., Rodríguez, R.: Finite element computation of threedimensional elastoacoustic vibrations. J. Sound Vibration, 219, 279-306 (1999).

16. Bermúdez, A., Rodríguez, R.: Finite element computation of the vibration modes of a fluid-solid system. Comput. Methods Appl. Mech. Engrg. 119, 355-370 (1994).

17. Boffi, D.: Finite element approximation of eigenvalue problems. Acta Numerica, 19, 1-120 (2010).

18. Boffi, D., Gardini, F., Gastaldi, L.: Some remarks on eigenvalue approximation by finite elements. In: Frontiers in Numerical Analysis-Durham 2010. Lect. Notes Comput. Sci. Eng. 85, Springer, Heidelberg, 1-77 (2012).

19. Brenner, S. C., Scott, R. L.: The Mathematical Theory of Finite Element Methods. Springer, New York (2008).

20. Brezzi, F., Falk, R. S., Marini, L. D.: Basic principles of mixed virtual element methods. ESAIM Math. Model. Numer. Anal. 48, 1227-1240 (2014).

21. Brezzi, F., Marini, L. D.: Virtual elements for plate bending problems. Comput. Methods Appl. Mech. Engrg. 253, 455-462 (2012).

22. Cangiani, A., Georgoulis, E. H., Houston, P.: $h p$-version discontinuous Galerkin methods on polygonal and polyhedral meshes. Math. Models Methods Appl. Sci. 24, 2009-2041 (2014).

23. Ciarlet, P. G.: The Finite Element Method for Elliptic Problems. SIAM, Philadelphia (2002).

24. Descloux, J., Nassif, N., Rappaz, J.: On spectral approximation. Part 1: The problem of convergence. RAIRO Anal. Numér. 12, 97-112 (1978).

25. Descloux, J., Nassif, N., Rappaz, J.: On spectral approximation. Part 2: Error estimates for the Galerkin method. RAIRO Anal. Numér. 12, 113-119 (1978).

26. Di Pietro, D., Ern, A.: A hybrid high-order locking-free method for linear elasticity on general meshes. Comput. Methods Appl. Mech. Eng. 283, 1-21 (2015). 
27. Di Pietro, D., Ern, A.: Hybrid high-order methods for variable-diffusion problems on general meshes. C. R. Acad. Sci., Paris I, 353, 31-34 (2015).

28. Gain, A. L., Talischi, C., Paulino, G.H.: On the virtual element method for three-dimensional linear elasticity problems on arbitrary polyhedral meshes. Comput. Methods Appl. Mech. Engrg. 282, 132-160 (2014).

29. Hamdi, M., Ousset, Y.and Verchery, G.: A displacement method for the analysis of vibrations of coupled fluid-structure systems. Internat. J. Numer. Methods Engrg. 13, 139-150 (1978).

30. Kiefling, L., Feng, G. C.: Fluid-structure finite element vibrational analysis. AIAA J. 14, 199203 (1976).

31. Lovadina, C., Mora, D., Rodríguez, R.: Approximation of the buckling problem for ReissnerMindlin plates. SIAM J. Numer. Anal. 48, 603-632 (2010).

32. Mora, D., Rivera, G., Rodríguez, R.: A virtual element method for the Steklov eigenvalue problem. Math. Models Methods Appl. Sci. 25, 1421-1445 (2015).

33. Perugia, I., Pietra, P., Russo, A.: A plane wave virtual element method for the Helmholtz problem. Preprint arXiv:1505.04965 [math.NA](2015).

34. Rodríguez, R., Solomin, J.: The order of convergence of eigenfrequencies in finite element approximations of fluid-structure interaction problems. Math. Comp. 65, 1463-1475 (1996).

35. Sukumar, N., Tabarraei, A.: Conforming polygonal finite elements. Internat. J. Numer. Methods Engrg. 61, 2045-2066 (2004).

36. Talischi, C., Paulino, G. H., Pereira, A., Menezes, I. F. M.: Polygonal finite elements for topology optimization: A unifying paradigm. Internat. J. Numer. Methods Engrg. 82, 671698 (2010).

37. Zienkiewicz, O. C., Taylor, R. L.: The Finite Element Method. Vol. 2, McGraw-Hill, London, 1991. 